\title{
Searching for dynamical black holes in various theories of gravity
}

\author{
Shin'ichi Nojiri, ${ }^{1,2}$ Sergei D. Odintsov, ${ }^{3,4,5,6}$ and Valerio Faraoni $\odot^{7}$ \\ ${ }^{1}$ Department of Physics, Nagoya University, Nagoya 464-8602, Japan \\ ${ }^{2}$ Kobayashi-Maskawa Institute for the Origin of Particles and the Universe, Nagoya University, \\ Nagoya 464-8602, Japan \\ ${ }^{3}$ Institució Catalana de Recerca i Estudis Avanćats (ICREA), Passeig Lluís Companys, \\ 23, 08010 Barcelona, Spain \\ ${ }^{4}$ Institute of Space Sciences (ICE, CSIC) C. Can Magrans s/n, 08193 Barcelona, Spain \\ ${ }^{5}$ Institute of Space Sciences of Catalonia (IEEC), Barcelona, Spain \\ ${ }^{6}$ Laboratory for Theoretical Cosmology, TUSUR, 634050 Tomsk and TSPU, 634061 Tomsk, Russia \\ ${ }^{7}$ Department of Physics and Astronomy, Bishop's University, \\ 2600 College Street, Sherbrooke, Québec, Canada J1M $1 Z 7$
}

(Received 22 October 2020; accepted 7 February 2021; published 25 February 2021)

\begin{abstract}
We construct models of Einstein and $f(R)$ gravity with two scalar fields, which admit analytical solutions describing time-varying dynamical black holes. Their thermodynamics is investigated in the adiabatic approximation. In addition to the Misner-Sharp-Hernandez quasilocal mass, we provide timedependent thermodynamical quantities, including the Hawking temperature, Helmholtz free energy, entropy, and thermodynamical energy. The latter does not always coincide with the Misner-SharpHernandez mass at the horizon, although they coincide in the static limit. For Schwarzschild-type (i.e., $g_{t t} g_{r r}=-1$ ) black holes in Einstein gravity, one of the two scalars is always a ghost with negative kinetic energy. We show that this ghost can be avoided in $f(R)$ gravity.
\end{abstract}

DOI: 10.1103/PhysRevD.103.044055

\section{INTRODUCTION}

Black hole dynamics and thermodynamics, developed for stationary and asymptotically flat black holes (e.g., [1-3]), are now mature fields of gravitational physics. However, no black hole is truly stationary or asymptotically flat. From the astrophysical point of view, realistic black holes interact with their environments in binary systems or in galaxies, through tidal forces, by accreting gas, and/or by emitting gravitational waves. From the purely theoretical point of view, black holes emit Hawking radiation losing energy, and are embedded in the expanding universe instead of being truly isolated, asymptotically flat, systems. Therefore, the ultimate theoretical description of black holes requires the consideration of dynamical solutions of the gravitational field equations. This is not a small step, both conceptually and computationally. Black holes are defined by their horizons: for stationary black holes, these are event horizons and null surfaces and their definition as a connected component of the boundary of the causal past of future null infinity [1] requires the knowledge of the entire causal structure (including the future history) of spacetime, which is summarized by saying that event horizons are teleological [4-7]. This traditional black hole concept becomes essentially useless for practical purposes in dynamical situations and is replaced by the more useful quasilocal definition of trapping surfaces and apparent or trapping horizons. Being defined quasilocally, apparent horizons require only the knowledge of a limited portion of spacetime [4-8]. In spite of some disadvantages (most notably, a dependence on the spacetime foliation $[9,10]$, and the fact that they are spacelike or timelike surfaces and that they can change their causal character during their dynamical evolution), apparent horizons are widely used in numerical simulations of the collisions of black holes with other compact objects that led to the interferometric discovery of gravitational waves [11-13]. Given the need to predict in detail the waveforms of gravitational waves emitted by binary systems containing black holes, banks of templates for gravitational waveforms need to be built to separate signals from environmental and other noise in the laser interferometric detectors of gravitational waves. The notion of event horizon is of little use in the numerical study of fast astrophysical processes producing those gravitational waves. Instead, "black holes" are routinely identified with outermost marginally trapped surfaces and apparent horizons in numerical research [4-7]. Numerical relativity uses apparent/trapping horizons, not event horizons.

Given this relatively recent paradigm shift, it would be useful to have a catalog of analytical solutions of general relativity (GR) and alternative theories of gravity describing dynamical black holes, and defined by their apparent horizons. Contrary to static and stationary black holes, very 
few dynamical black hole solutions are known in GR, and even fewer in alternative theories of gravity. Finding (and interpreting) new time-dependent exact solutions describing time-varying black holes is a rather difficult task already in GR. In principle, approaching this task in the context of alternative theories of gravity, which provide more gravitational degrees of freedom and, therefore, more flexibility, should be easier, but this does not seem to be the case.

From the point of view of black hole thermodynamics, placing a black hole in a nontrivial environment changes its mass-energy, which plays the role of internal energy in the first law of black hole thermodynamics. This mass-energy is best defined quasilocally. Here we set out to find analytical solutions of various theories of gravity and, for simplicity, we restrict ourselves to spherical symmetry, which has the added advantage that apparent horizons coincide in all spherical foliations [14]. In this case the mass-energy of a spherical geometry, asymptotically flat or not, is the MisnerSharp-Hernandez mass universally used in relativistic fluid dynamics and in studies of black hole collapse $[15,16]$ and which coincides, in spherical symmetry, with the HawkingHayward quasilocal energy $[17,18]$.

A few spherically symmetric solutions interpreted as genuine black holes embedded in Friedmann-LemaîtreRobertson-Walker (FLRW) universes are known in GR, beginning with the McVittie family ${ }^{1}$ [19], which has been generalized [20,21] and has been the subject of much attention during the last decade [22-38], also in the context of Hořava-Lifschitz and Horndeski gravity [39,40]. The phenomenology of apparent horizons can be rather bizarre and provides several surprises, such as horizons appearing and disappearing in pairs, as in the Husain-Martinez-Nuñez solution (a black hole embedded in a FLRW universe sourced by a free scalar field) [41]. The nonrotating Thakurta solution [42] is a late-time attractor of the generalized McVittie solutions [43]. Other solutions of Einstein's theory are less significant because they suffer from negative energy densities or instabilities in certain spacetime regions [44-48], while attempts to build new solutions have had mixed success [49-64].

Dynamical black hole solutions of alternative theories of gravity include the Clifton geometry in $f(R)=R^{n}$ gravity $[65,66]$ and some members of the Clifton-Mota-Barrow family in Brans-Dicke gravity with a perfect fluid $[67,68]$.
Since many alternative theories of gravity contain effective scalar degrees of freedom of gravitational origin, in spherical symmetry scalars either collapse to the Schwarzschild black hole [69-72] or tend to generate naked singularities or wormhole throats. It is not surprising that research in alternative theories produces these exotic objects more often than new black holes (e.g., [73-86]). Here we dismiss these exotica and we look for dynamical black holes, characterized by time-varying apparent horizons, in various theories of gravity. We follow the notation and conventions of Ref. [1], using units in which the speed of light $c$ and Newton's constant $G$ are unity, while $\kappa^{2} \equiv 8 \pi G$.

\section{SPHERICALLY SYMMETRIC AND TIME-DEPENDENT GEOMETRIES}

The most general spherically symmetric and timedependent line element in polar coordinates $(\tau, \rho, \vartheta, \varphi)$ is

$$
\begin{aligned}
d s^{2}= & -\mathcal{A}(\tau, \rho) d \tau^{2}+2 \mathcal{B}(\tau, \rho) d \tau d \rho+\mathcal{C}(\tau, \rho) d \rho^{2} \\
& +\mathcal{D}(\tau, \rho)\left(d \vartheta^{2}+\sin ^{2} \vartheta d \varphi^{2}\right),
\end{aligned}
$$

where the areal radius $r$ is defined by

$$
r^{2} \equiv \mathcal{D}(\tau, \rho)
$$

and $\mathcal{D}(\tau, \rho)$ (as well as $\mathcal{A}$ and $\mathcal{C}$ ) is necessarily positive to preserve the metric signature -+++ . In principle, Eq. (2.2) can be solved for $\rho(\tau, r)$ (although, in practice, it may be difficult to invert explicitly the one-to-one relation $r=$ $\sqrt{D(\tau, \rho)})$. In terms of the areal radius, the line element (2.1) is rewritten as

$$
\begin{aligned}
d s^{2}= & {\left[-\mathcal{A}(\tau, \rho(\tau, r))+2 \mathcal{B}(\tau, \rho(\tau, r)) \frac{\partial \rho}{\partial \tau}\right] d \tau^{2} } \\
& +2 \mathcal{B}(\tau, \rho(\tau, r)) \frac{\partial \rho}{\partial r} d \tau d r+\mathcal{C}(\tau, \rho(\tau, r))\left(\frac{\partial \rho}{\partial r}\right)^{2} d r^{2} \\
& +r^{2}\left(d \vartheta^{2}+\sin ^{2} \vartheta d \varphi^{2}\right)
\end{aligned}
$$

In order to diagonalize this line element, we introduce a new time coordinate $t$. Substituting $\tau=\tau(t, r)$ into Eq. (2.3), the line element becomes

$$
\begin{aligned}
d s^{2}= & {\left[-\mathcal{A}(\tau(t, r), \rho(\tau(t, r), r))+2 \mathcal{B}(\tau(t, r), \rho(\tau(t, r), r)) \frac{\partial \rho(\tau(t, r), r)}{\partial \tau}\right]\left(\frac{\partial \tau(t, r)}{\partial t}\right)^{2} d t^{2} } \\
& +2\left[\mathcal{B}(\tau(t, r), \rho(\tau(t, r), r)) \frac{\partial \rho(\tau(t, r), r)}{\partial r} \frac{\partial \tau(t, r)}{\partial t}\right.
\end{aligned}
$$

\footnotetext{
${ }^{1}$ The well-known Schwarzschild-deSitter/Kottler geometry, which is a special case of the McVittie, is instead locally static.
} 


$$
\begin{aligned}
& \left.+\left\{-\mathcal{A}(\tau(t, r), \rho(\tau(t, r), r))+2 \mathcal{B}(\tau(t, r), \rho(\tau(t, r), r)) \frac{\partial \rho(\tau(t, r), r)}{\partial \tau}\right\} \frac{\partial \tau(t, r)}{\partial t} \frac{\partial \tau(t, r)}{\partial r}\right] d t d r \\
& +\left[\mathcal{C}(\tau, \rho(\tau, r))\left(\frac{\partial \rho}{\partial r}\right)^{2}+\mathcal{B}(\tau(t, r), \rho(t, r), r) \frac{\partial \rho(\tau(t, r), r)}{\partial r} \frac{\partial \tau(t, r)}{\partial r}\right. \\
& +\left\{-\mathcal{A}(\tau(t, r), \rho(\tau(t, r), r))+2 \mathcal{B}(\tau(t, r), \rho(\tau(t, r), r)) \frac{\partial \rho(\tau(t, r), r)}{\partial \tau}\right\} \\
& \left.\times\left(\frac{\partial \tau(t, r)}{\partial r}\right)^{2}\right] d r^{2}+r^{2}\left(d \vartheta^{2}+\sin ^{2} \vartheta d \varphi^{2}\right) .
\end{aligned}
$$

By choosing the new time coordinate $t$ so that

$$
\begin{aligned}
0= & \mathcal{B}(\tau(t, r), \rho(\tau(t, r), r)) \frac{\partial \rho(\tau(t, r), r)}{\partial r} \frac{\partial \tau(t, r)}{\partial t} \\
& +\left[-\mathcal{A}(\tau(t, r), \rho(\tau(t, r), r))+2 \mathcal{B}(\tau(t, r), \rho(\tau(t, r), r)) \frac{\partial \rho(\tau(t, r), r)}{\partial \tau}\right] \frac{\partial \tau(t, r)}{\partial t} \frac{\partial \tau(t, r)}{\partial r}
\end{aligned}
$$

the line element assumes the diagonal form

$$
d s^{2}=-\mathrm{e}^{2 \nu(t, r)} d t^{2}+\mathrm{e}^{2 \lambda(t, r)} d r^{2}+r^{2}\left(d \vartheta^{2}+\sin ^{2} \vartheta d \varphi^{2}\right),
$$

where

$$
\begin{aligned}
-\mathrm{e}^{2 \nu(t, r)} & \equiv\left\{-\mathcal{A}(\tau(t, r), \rho(\tau(t, r), r))+2 \mathcal{B}(\tau(t, r), \rho(\tau(t, r), r)) \frac{\partial \rho(\tau(t, r), r)}{\partial \tau}\right\}\left(\frac{\partial \tau(t, r)}{\partial t}\right)^{2} \\
\mathrm{e}^{2 \lambda(t, r)} \equiv & \mathcal{C}(\tau, \rho(\tau, r))\left(\frac{\partial \rho}{\partial r}\right)^{2}+\mathcal{B}(\tau(t, r), \rho(\tau(t, r), r)) \frac{\partial \rho(\tau(t, r), r)}{\partial r} \frac{\partial \tau(t, r)}{\partial r} \\
& +\left[-\mathcal{A}(\tau(t, r), \rho(\tau(t, r), r))+2 \mathcal{B}(\tau(t, r), \rho(\tau(t, r), r)) \frac{\partial \rho(\tau(t, r), r)}{\partial \tau}\right]\left(\frac{\partial \tau(t, r)}{\partial r}\right)^{2}
\end{aligned}
$$

We define the metric $\bar{g}_{i j}$ of the unit 2-sphere by $\sum_{i, j=1,2} \bar{g}_{i j} d x^{i} d x^{j}=d \vartheta^{2}+\sin ^{2} \vartheta d \varphi^{2}$. For the metric (2.6), the only nonvanishing connection coefficients are

$$
\begin{aligned}
& \Gamma_{t t}^{t}=\dot{\nu}, \quad \Gamma_{t t}^{r}=\mathrm{e}^{-2(\lambda-\nu)} \nu^{\prime}, \quad \Gamma_{t r}^{t}=\Gamma_{r t}^{t}=\nu^{\prime}, \\
& \Gamma_{r r}^{t}=\mathrm{e}^{2 \lambda-2 \nu} \dot{\lambda}, \quad \Gamma_{t r}^{r}=\Gamma_{r t}^{r}=\dot{\lambda}, \\
& \Gamma_{r r}^{r}=\lambda^{\prime}, \quad \Gamma_{j k}^{i}=\bar{\Gamma}_{j k}^{i}, \quad \Gamma_{i j}^{r}=-\mathrm{e}^{-2 \lambda} r \bar{g}_{i j}, \\
& \Gamma_{r j}^{i}=\Gamma_{j r}^{i}=\frac{1}{r} \delta_{j}^{i},
\end{aligned}
$$

where $\bar{\Gamma}_{j k}^{i}$ is the metric connection of $\bar{g}_{i j}$, while an overdot and a prime denote differentiation with respect to $t$ and $r$, respectively. Using the expression of the Riemann tensor [1]

$$
R_{\mu \rho \nu}^{\lambda}=\Gamma_{\mu \nu, \rho}^{\lambda}-\Gamma_{\mu \rho, \nu}^{\lambda}+\Gamma_{\mu \nu}^{\eta} \Gamma_{\rho \eta}^{\lambda}-\Gamma_{\mu \rho}^{\eta} \Gamma_{\nu \eta}^{\lambda},
$$

one finds

$$
\begin{aligned}
& R_{r t r t}=-\mathrm{e}^{2 \lambda}[\ddot{\lambda}+(\dot{\lambda}-\dot{\nu}) \dot{\lambda}]+\mathrm{e}^{2 \nu}\left[\nu^{\prime \prime}+\left(\nu^{\prime}-\lambda^{\prime}\right) \nu^{\prime}\right], \\
& R_{t i t j}=r \nu^{\prime} \mathrm{e}^{2(\nu-\lambda)} \bar{g}_{i j}, \\
& R_{\text {rirj }}=\lambda^{\prime} r \bar{g}_{i j}, \quad R_{t i r j}=\dot{\lambda} r \bar{g}_{i j}, \quad R_{i j k l}=\left(1-\mathrm{e}^{-2 \lambda}\right) r^{2}\left(\bar{g}_{i k} \bar{g}_{j l}-\bar{g}_{i l} \bar{g}_{j k}\right),
\end{aligned}
$$




$$
\begin{aligned}
R_{t t} & =-[\ddot{\lambda}+(\dot{\lambda}-\dot{\nu}) \dot{\lambda}]+\mathrm{e}^{2(\nu-\lambda)}\left[\nu^{\prime \prime}+\left(\nu^{\prime}-\lambda^{\prime}\right) \nu^{\prime}+\frac{2 \nu^{\prime}}{r}\right], \\
R_{r r} & =\mathrm{e}^{-2(\nu-\lambda)}[\ddot{\lambda}+(\dot{\lambda}-\dot{\nu}) \dot{\lambda}]-\left[\nu^{\prime \prime}+\left(\nu^{\prime}-\lambda^{\prime}\right) \nu^{\prime}\right]+\frac{2 \lambda^{\prime}}{r}, \\
R_{i j} & =\left[1+\left\{-1-r\left(\nu^{\prime}-\lambda^{\prime}\right)\right\} \mathrm{e}^{-2 \lambda}\right] \bar{g}_{i j}, \\
R & =2 \mathrm{e}^{-2 \nu}[\ddot{\lambda}+(\dot{\lambda}-\dot{\nu}) \dot{\lambda}]+\mathrm{e}^{-2 \lambda}\left[-2 \nu^{\prime \prime}-2\left(\nu^{\prime}-\lambda^{\prime}\right) \nu^{\prime}-\frac{4\left(\nu^{\prime}-\lambda^{\prime}\right)}{r}+\frac{2 \mathrm{e}^{2 \lambda}-2}{r^{2}}\right] .
\end{aligned}
$$

Any spherically symmetric metric can be recast in the Abreu-Nielsen-Visser gauge [87,88]

$$
\begin{aligned}
d s^{2}= & -\mathrm{e}^{-2 \Phi(t, r)}\left(1-\frac{2 M_{\mathrm{MSH}}(t, r)}{r}\right) d t^{2}+\frac{d r^{2}}{1-\frac{2 M_{\mathrm{MSH}}(t, r)}{r}} \\
& +r^{2}\left(d \vartheta^{2}+\sin ^{2} \vartheta d \varphi^{2}\right)
\end{aligned}
$$

where $r$ is the areal radius and $M_{\mathrm{MSH}}$ is the Misner-SharpHernandez mass defined in spherical symmetry by $[15,16]$

$$
1-\frac{2 M_{\mathrm{MSH}}}{r}=\nabla^{c} r \nabla_{c} r
$$

which, in the gauge (2.12), reduces to $g^{r r}=1-2 M_{\mathrm{MSH}} / r$. (Although this definition was originally given in GR $[15,16]$, it applies to other theories of gravity as well, but its role in the relevant equations may change [89,90].) The more general Hawking-Hayward quasilocal mass $[17,18]$ reduces to the Misner-Sharp-Hernandez mass in spherical symmetry [91] and is not restricted to asymptotically flat spacetimes (however, in the asymptotically flat case, the Hawking-Hayward/Misner-Sharp-Hernandez mass computed at spatial infinity reduces to the ArnowittDeser-Misner (ADM) mass [92]).

By comparing the Abreu-Nielsen-Visser gauge (2.12) with Eq. (2.6), one has the correspondence

$$
\begin{gathered}
\mathrm{e}^{2 \nu}=\mathrm{e}^{-2 \Phi}\left(1-\frac{2 M_{\mathrm{MSH}}}{r}\right)=\mathrm{e}^{-2(\lambda+\Phi)}, \\
\mathrm{e}^{2 \lambda}=\left(1-\frac{2 M_{\mathrm{MSH}}}{r}\right)^{-1} .
\end{gathered}
$$

We are interested in apparent horizons, which can be dynamical, and are located by the roots of $\nabla^{c} r \nabla_{c} r=0$, or $r_{\mathrm{AH}}=2 M_{\mathrm{MSH}}\left(r_{\mathrm{AH}}\right)[8,15,16,88]$.

\section{SPHERICALLY SYMMETRIC AND TIME- DEPENDENT SOLUTIONS OF EINSTEIN- TWO-SCALAR MODELS}

Let us first consider GR with two scalar fields $\phi$ and $\chi$ as the matter source, as described by the action

$$
S_{(\mathrm{GR} \phi \chi)}=\int d^{4} x \sqrt{-g}\left[\frac{R}{2 \kappa^{2}}-\frac{1}{2} A(\phi, \chi) \partial_{\mu} \phi \partial^{\mu} \phi-B(\phi, \chi) \partial_{\mu} \phi \partial^{\mu} \chi-\frac{1}{2} C(\phi, \chi) \partial_{\mu} \chi \partial^{\mu} \chi-V(\phi, \chi)\right]
$$

where $g$ is the determinant of the metric tensor $g_{\mu \nu}, R$ is the Ricci scalar, $V(\phi, \chi)$ is the potential of the scalar doublet, and the coefficients $A, B$, and $C$ depend on the scalars. Scenarios with two or more scalar fields have been studied in the literature many times, especially in relation with multiple scalar field inflation (e.g., [93-98]), but there is no specific relation between those models and the theories that we use in the present manuscript. The matter energy-momentum tensor is

$$
\begin{aligned}
T_{\mu \nu}^{(\phi \chi)}= & g_{\mu \nu}\left[-\frac{1}{2} A(\phi, \chi) \partial_{\rho} \phi \partial^{\rho} \phi-B(\phi, \chi) \partial_{\rho} \phi \partial^{\rho} \chi-\frac{1}{2} C(\phi, \chi) \partial_{\rho} \chi \partial^{\rho} \chi-V(\phi, \chi)\right] \\
& +A(\phi, \chi) \partial_{\mu} \phi \partial_{\nu} \phi+B(\phi, \chi)\left(\partial_{\mu} \phi \partial_{\nu} \chi+\partial_{\nu} \phi \partial_{\mu} \chi\right)+C(\phi, \chi) \partial_{\mu} \chi \partial_{\nu} \chi
\end{aligned}
$$

and the contracted Bianchi identities read

$$
\begin{gathered}
0=\frac{A_{\phi}}{2} \partial_{\mu} \phi \partial^{\mu} \phi+A \nabla^{\mu} \partial_{\mu} \phi+A_{\chi} \partial_{\mu} \phi \partial^{\mu} \chi+\left(B_{\chi}-\frac{1}{2} C_{\phi}\right) \partial_{\mu} \chi \partial^{\mu} \chi+B \nabla^{\mu} \partial_{\mu} \chi-V_{\phi}, \\
0=\left(-\frac{1}{2} A_{\chi}+B_{\phi}\right) \partial_{\mu} \phi \partial^{\mu} \phi+B \nabla^{\mu} \partial_{\mu} \phi+\frac{1}{2} C_{\chi} \partial_{\mu} \chi \partial^{\mu} \chi+C \nabla^{\mu} \partial_{\mu} \chi+C_{\phi} \partial_{\mu} \phi \partial^{\mu} \chi-V_{\chi},
\end{gathered}
$$


where $A_{\phi} \equiv \partial A(\phi, \chi) / \partial \phi$, etc. We now identify

$$
\phi=t, \quad \chi=r .
$$

This assumption does not lead to a loss of generality for the following reason: for the spherically symmetric solutions (2.6) of the theory (3.1), in general $\phi$ and $\chi$ depend on both coordinates $t$ and $r$. Given a solution, the $t$ - and $r$ dependence of $\phi$ and $\chi$ can be determined and $\phi$ and $\chi$ are then given by specific functions $\phi(t, r), \chi(t, r)$. On spacetime regions where these relations are one-to-one, and provided that $\partial_{\mu} \phi$ is timelike and $\partial_{\mu} \chi$ is spacelike, one can invert these specific functional forms and redefine the scalar fields to replace $t$ and $r$ with new scalar fields, say, $\bar{\phi}$ and $\bar{\chi}$ with $\phi(t, r) \rightarrow \phi(\bar{\phi}, \bar{\chi})$ and $\chi(t, r) \rightarrow \chi(\bar{\phi}, \bar{\chi})$. We can then identify the new fields with $t$ and $r$ as in (3.5), $\bar{\phi} \rightarrow \phi=t$ and $\bar{\chi} \rightarrow \chi=r$. The change of variables $(\phi, \chi) \rightarrow(\bar{\phi}, \bar{\chi})$ can then be absorbed into redefinitions of $A, B, C$, and $V$ in the action (3.1). Therefore, the assumption (3.5) does not lead to loss of generality.

Proceeding, the $(t, t),(r, r),(i, j)$, and $(t, r)$ components of the Einstein equations assume, respectively, the form

$\frac{\mathrm{e}^{2(\nu-\lambda)}}{\kappa^{2}}\left(\frac{2 \lambda^{\prime}}{r}+\frac{\mathrm{e}^{2 \lambda}-1}{r^{2}}\right)=-\mathrm{e}^{2 \nu}\left(-\frac{A}{2} \mathrm{e}^{-2 \nu}-\frac{C}{2} \mathrm{e}^{-2 \lambda}-V\right)$,

$\frac{1}{\kappa^{2}}\left(\frac{2 \nu^{\prime}}{r}-\frac{\mathrm{e}^{2 \lambda}-1}{r^{2}}\right)=\mathrm{e}^{2 \lambda}\left(\frac{A}{2} \mathrm{e}^{-2 \nu}+\frac{C}{2} \mathrm{e}^{-2 \lambda}-V\right)$,

$$
\begin{aligned}
& \frac{1}{\kappa^{2}}\left[-\mathrm{e}^{-2 \nu}\{\ddot{\lambda}+(\dot{\lambda}-\dot{\nu}) \dot{\lambda}\}+\mathrm{e}^{-2 \lambda}\left(r\left(\nu^{\prime}-\lambda^{\prime}\right)\right.\right. \\
& \left.\left.+r^{2} \nu^{\prime \prime}+r^{2}\left(\nu^{\prime}-\lambda^{\prime}\right) \nu^{\prime}\right)\right]=r^{2}\left(\frac{A}{2} \mathrm{e}^{-2 \nu}-\frac{C}{2} \mathrm{e}^{-2 \lambda}-V\right),
\end{aligned}
$$

$$
\frac{2 \dot{\lambda}}{\kappa^{2} r}=B
$$

Due to spherical symmetry, the other components of the Einstein equation are trivially satisfied. Equations (3.6)(3.9) can be solved with respect to $A, B, C$, and $V$, obtaining the inverse relations

$$
\begin{gathered}
A=\frac{1}{\kappa^{2}}[-\{\ddot{\lambda}+(\dot{\lambda}-\dot{\nu}) \dot{\lambda}\} \\
\left.+\mathrm{e}^{2(\nu-\lambda)}\left(\frac{\mathrm{e}^{2 \lambda}-1}{r^{2}}+\frac{\nu^{\prime}+\lambda^{\prime}}{r}+\nu^{\prime \prime}+\left(\nu^{\prime}-\lambda^{\prime}\right) \nu^{\prime}\right)\right], \\
B=\frac{2 \dot{\lambda}}{\kappa^{2} r},
\end{gathered}
$$

$$
\begin{array}{r}
C=\frac{1}{\kappa^{2}}\left[\frac{\mathrm{e}^{-2(\nu-\lambda)}}{r^{2}}\{\ddot{\lambda}+(\dot{\lambda}-\dot{\nu}) \dot{\lambda}\}-\frac{\mathrm{e}^{2 \lambda}-1}{r^{2}}\right. \\
\left.+\frac{\nu^{\prime}+\lambda^{\prime}}{r}-\nu^{\prime \prime}-\left(\nu^{\prime}-\lambda^{\prime}\right) \nu^{\prime}\right], \\
V=\frac{\mathrm{e}^{-2 \lambda}}{2 \kappa^{2}}\left[\frac{2\left(\lambda^{\prime}-\nu^{\prime}\right)}{r}+\frac{2\left(\mathrm{e}^{2 \lambda}-1\right)}{r^{2}}\right] .
\end{array}
$$

The right-hand sides of Eqs. (3.10)-(3.13) are functions of $t$ and $r$. Replacing $(t, r)$ with $(\phi, \chi)$ in these right-hand sides, we obtain $A, B, C$, and $V$ as functions of $(\phi, \chi)$. Conversely, if we prescribe $A, B, C$, and $V$, the model has as solutions spherically symmetric configurations (2.6) corresponding to arbitrary functions $\nu$ and $\lambda$.

We may also consider solutions for which $\lambda=-\nu$, as in the Schwarzschild geometry. In a spherical spacetime in which the line element is written using the areal radius $r$ and $g_{t t} g_{r r}=-1, r$ is an affine parameter along radial null geodesics [99]. Furthermore, such a spacetime enjoys special algebraic properties ([99], see also [100,101]): the double projection $R_{\mu \nu} l^{\mu} l^{\nu}$ of the Ricci tensor onto radial null vectors $l^{\alpha}$ vanishes identically [99]. Alternatively, the restriction of the Ricci tensor to the $(t, r)$ submanifold is proportional to the restriction of the metric $g_{\mu \nu}$ to this subspace [99]. Many analytical solutions of interest in GR or in alternative gravities satisfy the condition $g_{t t} g_{r r}=-1$, including vacuum solutions, electrovacuum solutions with the Maxwell or with nonlinear Born-Infeld electrodynamics [99], and the string hedgehog global monopole [102,103], also in higher dimension.

Under the condition $\lambda=-\nu$, Eqs. (3.10)-(3.13) simplify to

$$
\begin{gathered}
A=-r^{2} \mathrm{e}^{4 \nu} C \\
=\frac{1}{\kappa^{2}}\left[-\frac{\mathrm{e}^{2 \nu}}{2} \frac{d^{2}\left(\mathrm{e}^{-2 \nu}\right)}{d t^{2}}+\mathrm{e}^{4 \nu}\left(\frac{\mathrm{e}^{-2 \nu}-1}{r^{2}}+\frac{\mathrm{e}^{-2 \nu}}{2} \frac{d^{2}\left(\mathrm{e}^{2 \nu}\right)}{d r^{2}}\right)\right] \\
B=\frac{\mathrm{e}^{2 \nu}}{\kappa^{2} r} \frac{d\left(\mathrm{e}^{-2 \nu}\right)}{d t} \\
V=\frac{\mathrm{e}^{2 \nu}}{2 \kappa^{2}}\left(-\frac{2 \mathrm{e}^{-2 \nu}}{r} \frac{d\left(\mathrm{e}^{2 \nu}\right)}{d r}+\frac{2\left(\mathrm{e}^{-2 \nu}-1\right)}{r^{2}}\right)
\end{gathered}
$$

Since $A C=-r^{2} \mathrm{e}^{4 \nu} C^{2}<0$, either $\phi$ or $\chi$ has negative kinetic energy in the action (3.1) and a ghost is always present in this case.

We may further restrict ourselves to the static case, $\nu=\nu(r)$ and $\lambda=\lambda(r)$; then, Eqs. (3.14) and (3.15) tell us that $A, B$, and $C$ depend only on $\chi$ and not on $\phi$. However, even in the static case, we still need $\phi$ to obtain a model corresponding to arbitrary $\nu$ and $\lambda$. 


\section{A. Ansatz 1}

As an example, consider the ansatz

$$
\mathrm{e}^{2 \nu}=\mathrm{e}^{-2 \lambda}=1-\frac{2 M(t)}{r},
$$

where the Misner-Sharp-Hernandez mass $M(t)$ is positive and depends only on time (clearly, a negative $M_{\mathrm{MSH}}$ signals a violation of the energy conditions and makes apparent horizons impossible). The Ricci curvature is

$$
R=\left(1-\frac{2 M}{r}\right)^{-1}\left[\frac{2 \ddot{M}}{r}+\frac{\left(\frac{2 \dot{M}}{r}\right)^{2}}{1-\frac{2 M}{r}}\right]
$$

and it vanishes if $M$ is constant, in which case the geometry degenerates into the Schwarzschild one. There is only one apparent horizon, with areal radius $r_{\mathrm{AH}}(t)=2 M(t)$. This horizon is dynamical and, since it is a single root of the equation $\nabla^{c} r \nabla_{c} r=0$, it is a black hole horizon. [A curvature singularity corresponds to $2 M / r=1$ if $\frac{2 \ddot{M}}{r}\left(1-\frac{2 M}{r}\right)+\left(\frac{2 \dot{M}}{r}\right)^{2}$ vanishes as $\mathcal{O}\left(\left(1-\frac{2 M}{r}\right)^{2}\right)$ when $2 M / r=1$, which is impossible.]

To discuss the thermodynamics of the spacetime (3.17), we assume that the time evolution is sufficiently slow $(\dot{M} \ll 1)$ and we regard the time $t$ as a constant for the purposes of thermodynamics. This adiabatic approximation is necessary in order to avoid dealing with full nonequilibrium thermodynamics, which is essentially unknown for dynamical black holes [8]. It is present (although often not made explicit) in the tunneling formalism for dynamical black holes (see [104] for a review). In practice, it amounts to requiring that the horizon moves "slowly," or that the time derivatives of the functions used in the thermodynamical calculations are much smaller than the corresponding spatial derivatives (the Kodama time is used in the tunneling method, but other gauges are in principle possible). In the adiabatic regime, the temperature is

$$
T=\frac{1}{8 \pi M(t)},
$$

and the Bekenstein-Hawking entropy reads

$$
\mathcal{S}=\frac{4 \pi\left[r_{\mathrm{AH}}(t)\right]^{2}}{4}=\frac{1}{16 \pi T^{2}} .
$$

Then, since $d F / d T=-\mathcal{S}$, where $F$ is the free energy, we have

$$
F=\frac{1}{16 \pi T}
$$

by choosing the integration constant so that $F \rightarrow 0$ in the formal limit $T \rightarrow \infty$ (or $M(t) \rightarrow 0$ ). The thermal energy is

$$
E=F+T \mathcal{S}=\frac{1}{8 \pi T}=M(t) .
$$

If $M$ is constant, the expressions of $T, \mathcal{S}$, and $E$ reduce to those of the Schwarzschild black hole. We also find

$A=-\frac{1}{2 \kappa^{2}}\left[\frac{2 M^{\prime \prime}(\phi)}{\chi}+\frac{\left(\frac{2 M^{\prime}(\phi)}{\chi}\right)^{2}}{1-\frac{2 M(\phi)}{\chi}}\right]$,

$B=\frac{\frac{2 M^{\prime}(\phi)}{\chi}}{\kappa^{2} r\left(1-\frac{2 M(\phi)}{\chi}\right)}$,

$C=\frac{1}{2 \kappa^{2} \chi^{2}\left(1-\frac{2 M(\phi)}{\chi}\right)^{2}}\left[\frac{2 M^{\prime \prime}(\phi)}{\chi}+\frac{\left(\frac{2 M^{\prime}(\phi)}{\chi}\right)^{2}}{1-\frac{2 M(\phi)}{\chi}}\right]$,

$V=0$.

Since $A C<0$, one of the two free scalars $\phi$ or $\chi$ is always a ghost, which makes the theory physically inconsistent.

\section{B. Ansatz 2}

It is sometimes possible to find exact solutions by separating the time and space dependence in the metric coefficients (as done, for example, in [65,67]). As another example, consider the ansatz

$$
\mathrm{e}^{2 \nu}=\mathrm{e}^{-2 \lambda}=\left(1-\frac{r_{0}}{r}\right) \frac{t_{0}}{t},
$$

where $r_{0}$ and $t_{0}$ are positive constants. The Misner-SharpHernandez quasilocal mass is

$$
M_{\mathrm{MSH}}(t, r)=\frac{r}{2}\left[1-\left(1-\frac{r_{0}}{r}\right) \frac{t_{0}}{t}\right]
$$

and there is only one apparent horizon located by $r_{\mathrm{AH}}=2 M_{\mathrm{MSH}}\left(t, r_{\mathrm{AH}}\right)$, which gives $r=r_{0}$. Since this is a single root, we have a black hole apparent horizon. Remarkably, although the metric and the Misner-SharpHernandez mass ${ }^{2}$ (of a generic sphere of radius $r$ ) are time dependent, this apparent horizon has constant (areal) radius. This horizon is a null surface and an event horizon. In fact, if $h(r) \equiv r-r_{0}$, the horizon is the surface $h(r)=0$ with normal

$$
N_{\mu}=\left.\nabla_{\mu} h\right|_{r=r_{0}}=\delta_{\mu 1}
$$

which is a null vector:

$$
g_{\mu \nu} N^{\mu} N^{\nu}=\left.g^{11}\right|_{r=r_{0}}=\left.\left(1-\frac{r_{0}}{r}\right) \frac{t_{0}}{t}\right|_{r=r_{0}}=0 .
$$

\footnotetext{
${ }^{2}$ The Misner-Sharp-Hernandez mass at the horizon $M_{\mathrm{MSH}}\left(r_{0}\right)=$ $r_{0} / 2$ is time independent.
} 
We have, therefore, a static event horizon in a nonstationary spacetime: the question of whether such a horizon can exist was posed, and approached perturbatively, in Ref. [105]. Furthermore, since there is no timelike Killing vector, this event horizon is not a Killing horizon, which is of interest in relation with the strong rigidity theorem $[106,107]$ stating that the event horizon of a stationary black hole spacetime is a Killing horizon. This theorem is not violated here because it requires the matter stress-energy tensor to satisfy the weak energy condition while, as we shall see shortly, one of the two scalar fields is a phantom and violates it.

The Ricci curvature is

$$
R=\frac{2}{r^{2}}\left(1-\frac{t_{0}}{t}\right)
$$

This geometry has no spacetime singularities except for the usual one at $r=0$ and the big bang at $t=0$.

With regard to the thermodynamics of the spacetime (3.24), we assume again an adiabatic regime (which is possibile when $t$ is not close to zero and the metric changes relatively slowly); then the temperature and the BekensteinHawking entropy are (see Appendix A for details)

$$
T=\frac{t_{0}}{4 \pi t r_{0}},
$$

and

$$
\mathcal{S}=\frac{4 \pi r_{0}^{2}}{4}
$$

Even though the apparent horizon is static, together with its area $\mathcal{A}_{\mathrm{AH}}$ and the entropy $\mathcal{S}=\mathcal{A}_{\mathrm{AH}} / 4$, the Hawking temperature decreases monotonically with time, which is interpreted as an effect of the accretion of the scalar doublet onto the black hole. Indeed, $\dot{\lambda} \neq 0$ and, therefore, $B \neq 0$ [as follows from Eq. (3.2)] and the component $T_{t r}^{(\phi \chi)}$ of the stress-energy tensor of the scalar doublet is nonvanishing, signaling a nonzero radial energy flux onto the black hole. It is noteworthy that, although the metric varies in time [which causes the Hawking temperature to vary according to Eq. (3.29)], the apparent horizon does not change its location. We are not aware of a similar occurrence in previous literature.

Eliminating $r_{0}$ with Eq. (3.29) gives

$$
\mathcal{S}=\frac{t_{0}^{2}}{4 t^{2} T^{2}},
$$

and integrating $d F / d T=-\mathcal{S}$ yields the Helmholtz free energy

$$
F=\frac{t_{0}^{2}}{4 t^{2} T}
$$

and the thermal energy

$$
E=F+T \mathcal{S}=\frac{t_{0}^{2}}{2 t^{2} T}
$$

and we obtain thermodynamical quantities with nontrivial time dependence that obey the first law

$$
d E=-\frac{t_{0}^{2}}{2 t^{2} T^{2}} d T=T d S=d Q
$$

where $d Q$ is the heat transferred across the horizon.

We also find

$$
\begin{gathered}
A=-\left(1-\frac{r_{0}}{\chi}\right)^{2} \frac{t_{0}^{2} \chi^{2}}{\phi^{2}}, \\
B=\frac{1}{\kappa^{2} r t}, \\
C=\frac{1}{\kappa^{2}}\left(1-\frac{r_{0}}{\chi}\right) \frac{t_{0}^{2}}{\phi^{2}}\left(\frac{\phi}{t_{0} \chi^{2}}-\frac{1}{\chi^{2}}\right), \\
V=\frac{\mathrm{e}^{-2 \lambda}}{2 \kappa^{2}}\left[\frac{2\left(\lambda^{\prime}-\nu^{\prime}\right)}{r}+\frac{2\left(\mathrm{e}^{2 \lambda}-1\right)}{r^{2}}\right] .
\end{gathered}
$$

Again, it is $A C<0$ and $\phi$ or $\chi$ is always a ghost.

\section{Ansatz 3}

Our last example choice is

$$
\mathrm{e}^{2 \nu}=\mathrm{e}^{-2 \lambda}=\frac{1-\frac{r_{0}}{r}}{1+\frac{t r_{0}}{t_{0} r}} .
$$

The Misner-Sharp-Hernandez quasilocal mass is

$$
M_{\mathrm{MSH}}(t, r)=\frac{r_{0}}{2}\left(\frac{1+\frac{t}{t_{0}}}{1+\frac{t r_{0}}{t_{0} r}}\right)
$$

and the apparent horizons are located by $r=2 M_{\mathrm{MSH}}$, which yields the only root $r=r_{0}$. This is a single root and the radius of a static black hole event horizon. The mass at this horizon is $M_{\mathrm{MSH}}\left(r_{0}\right)=r_{0} / 2$ and does not depend on time.

Since $\mathrm{e}^{2 \nu}=\mathrm{e}^{-2 \lambda} \rightarrow 1$ as $r \rightarrow \infty$, the geometry is asymptotically flat.

In the limit $t \rightarrow \infty$ with fixed $r$, the metric has the form $\mathrm{e}^{2 \nu} \simeq \frac{t_{0} r}{t r_{0}}\left(1-\frac{r_{0}}{r}\right)$. Then, by introducing a new time coordinate $\tau$ defined by $d \tau=t^{-1 / 2} d t$ (or $\tau(t)=2 \sqrt{t}$ ), the line element (2.6) is recast in the form 


$$
\begin{aligned}
d s^{2}= & -\frac{t_{0} r}{r_{0}}\left(1-\frac{r_{0}}{r}\right) d \tau^{2}+\frac{r_{0} \tau^{2}}{4 t_{0} r\left(1-r_{0} / r\right)} d r^{2} \\
& +r^{2}\left(d \vartheta^{2}+\sin ^{2} \vartheta d \varphi^{2}\right),
\end{aligned}
$$

in which the radial direction becomes larger like a throat as the time $\tau$ (or $t$ ) increases. The areal radius of the horizon, however, remains constant. The time-dependent factor $\left(\frac{r_{0}}{4 t_{0} r\left(1-\frac{r_{0}}{r}\right)} \tau^{2}\right)$ multiplies only $d r^{2}$ and not the angular part of the line element, as would happen for a central object embedded in a FLRW universe [8] (indeed, the metric is asymptotically flat and not asymptotically FLRW).

The Ricci curvature of the geometry (3.39) is

$R=\frac{2\left(1+\frac{t_{0}}{t}\right) \frac{r_{0}}{r^{3}}}{\left(1+\frac{t r_{0}}{t_{0} r}\right)^{3}}\left[1-\frac{t}{t_{0}}+2\left(1-\frac{t}{t_{0}}\right) \frac{t r_{0}}{t_{0} r}+\frac{t^{2} r_{0}^{2}}{t_{0}^{2} r^{2}}\right]$

and is regular on the horizon $r=r_{0}$, although there are spacetime singularities at $r=0, t=0$, and $t \rightarrow \infty$.

With the ansatz (3.39), the Hawking temperature is

$$
T=\frac{1}{4 \pi r_{0}\left(1+t / t_{0}\right)}
$$

and, since the entropy $\mathcal{S}$ is

$$
\mathcal{S}=\frac{4 \pi r_{0}^{2}}{4}=\frac{1}{16 \pi\left(1+t / t_{0}\right)^{2} T^{2}}
$$

the free energy and the thermodynamical energy are

$$
F=\frac{1}{16 \pi\left(1+t / t_{0}\right)^{2} T}, \quad E=\frac{1}{8 \pi\left(1+t / t_{0}\right)^{2} T},
$$

respectively. Again, the black hole horizon is static while the Hawking temperature decreases with time (the adiabatic approximation can be satisfied by choosing $t_{0}$ and the range of $t$ appropriately).

We also have

$$
\begin{aligned}
A & =-\frac{\left(1-\frac{r_{0}}{r}\right)^{2} r^{2}}{\left(1+\frac{t 0_{0}}{t_{0} r}\right)^{2}} C \\
& =-\frac{\left(1-\frac{r_{0}}{r}\right)\left(1+\frac{t_{0}}{t}\right) t r_{0}}{\kappa^{2}\left(1+\frac{t r_{0}}{t_{0}}\right)^{2} t_{0} r^{3}}\left[1+\frac{1}{\left(1+\frac{t r_{0}}{t_{0} r}\right)^{2}}\right], \\
B & =\frac{r_{0}}{\kappa^{2} t_{0} r^{2}\left(1+\frac{t r_{0}}{t_{0} r}\right)}, \\
V & =\frac{\left(1+\frac{t_{0}}{t}\right) r_{0}}{\kappa^{2} r^{3}}\left[-\frac{\frac{t}{t_{0}}}{\left(1+\frac{t r_{0}}{t_{0} r}\right)^{2}}+\frac{1}{1+\frac{t r_{0}}{t_{0} r}}\right] .
\end{aligned}
$$

As in the case of Eqs. (3.46), (3.15), and (3.16), also this example is plagued by a phantom with negative kinetic energy because $A C<0$.

\section{IV. $f(R)$ GRAVITY WITH TWO SCALAR FIELDS}

The unavoidable recurrence of a phantom in Einstein's gravity with two scalar fields prompts us to investigate $f(R)$ gravity in the hope to exorcise this ghost. The equation of motion for this modified gravity is

$$
\begin{aligned}
f^{\prime}(R) R_{\mu \nu}-\frac{f(R)}{2} g_{\mu \nu}= & \nabla_{\mu} \nabla_{\nu} f^{\prime}(R)-g_{\mu \nu} \square f^{\prime}(R) \\
& +\kappa^{2} T_{\mu \nu}^{\text {(matter) }},
\end{aligned}
$$

where $T_{\mu \nu}^{(\text {matter })}$ is the matter energy-momentum tensor and $f^{\prime}(R) \equiv d f / d R$. Assuming the energy-momentum tensor (3.2) of the scalar field doublet, as in (3.6)-(3.9), the components of the field equation (4.1) become

$$
\begin{aligned}
&- \frac{\mathrm{e}^{2 \nu}}{2} f(R)-[-\{\ddot{\lambda}+(\dot{\lambda}-\dot{\nu}) \dot{\lambda}\} \\
&\left.+\mathrm{e}^{2(\nu-\lambda)}\left\{\nu^{\prime \prime}+\left(\nu^{\prime}-\lambda^{\prime}\right) \nu^{\prime}+\frac{2 \nu^{\prime}}{r}\right\}\right] f^{\prime}(R) \\
&+\left[-\dot{\lambda} \partial_{t}+\mathrm{e}^{2 \nu-2 \lambda}\left(\partial_{r}^{2}+\left(-\lambda^{\prime}+\frac{2}{r}\right) \partial_{r}\right)\right] f^{\prime}(R) \\
&= \mathrm{e}^{2 \nu}\left(-\frac{A}{2} \mathrm{e}^{-2 \nu}-\frac{C}{2} \mathrm{e}^{-2 \lambda}-V\right), \\
& \frac{\mathrm{e}^{2 \lambda}}{2} f(R)-\left[\mathrm{e}^{-2(\nu-\lambda)}\{\ddot{\lambda}+(\dot{\lambda}-\dot{\nu}) \dot{\lambda}\}\right. \\
&\left.\quad-\left\{\nu^{\prime \prime}+\left(\nu^{\prime}-\lambda^{\prime}\right) \nu^{\prime}\right\}+\frac{2 \lambda^{\prime}}{r}\right] f^{\prime}(R) \\
& \quad-\left[-\mathrm{e}^{2 \lambda-2 \nu}\left(\partial_{t}^{2}-\dot{\nu} \partial_{t}\right)+\left(\nu^{\prime}+\frac{2}{r}\right) \partial_{r}\right] f^{\prime}(R) \\
&=-\mathrm{e}^{2 \lambda}\left(\frac{A}{2} \mathrm{e}^{-2 \nu}+\frac{C}{2} \mathrm{e}^{-2 \lambda}-V\right),
\end{aligned}
$$

$$
\begin{aligned}
\frac{1}{2} f(R) & -\frac{1}{r^{2}}\left\{1+\left\{-1-r\left(\nu^{\prime}-\lambda^{\prime}\right)\right\} \mathrm{e}^{-2 \lambda}\right\} f^{\prime}(R) \\
- & {\left[-\mathrm{e}^{-2 \nu}\left(\partial_{t}^{2}+(-\dot{\nu}+\dot{\lambda}) \partial_{t}\right)\right.} \\
& \left.+\mathrm{e}^{-2 \lambda}\left(\partial_{r}^{2}+\left(\nu^{\prime}-\lambda^{\prime}+\frac{1}{r}\right) \partial_{r}\right)\right] f^{\prime}(R) \\
= & -\left(\frac{A}{2} \mathrm{e}^{-2 \nu}-\frac{C}{2} \mathrm{e}^{-2 \lambda}-V\right), \\
- & \frac{2 \dot{\lambda}}{r} f^{\prime}(R)+\left(\partial_{t} \partial_{r}-\nu^{\prime} \partial_{t}-\dot{\lambda} \partial_{r}\right) f^{\prime}(R)=-B .
\end{aligned}
$$

Then, we have 


$$
\begin{aligned}
A= & {\left[-\{\ddot{\lambda}+(\dot{\lambda}-\dot{\nu}) \dot{\lambda}\}+\mathrm{e}^{2(\nu-\lambda)}\left\{\nu^{\prime \prime}+\left(\nu^{\prime}-\lambda^{\prime}\right) \nu^{\prime}+\frac{2 \nu^{\prime}}{r}\right\}\right.} \\
& \left.-\frac{\mathrm{e}^{2 \nu}}{r^{2}}\left\{1+\left\{-1-r\left(\nu^{\prime}-\lambda^{\prime}\right)\right\} \mathrm{e}^{-2 \lambda}\right\}\right] f^{\prime}(R) \\
& -\left[-\partial_{t}^{2}+\dot{\nu} \partial_{t}+\mathrm{e}^{2 \nu-2 \lambda}\left(\nu^{\prime}+\frac{1}{r}\right) \partial_{r}\right] f^{\prime}(R) \\
C= & {\left[\mathrm{e}^{-2(\nu-\lambda)}[\ddot{\lambda}+(\dot{\lambda}-\dot{\nu}) \dot{\lambda}]-\left[\nu^{\prime \prime}+\left(\nu^{\prime}-\lambda^{\prime}\right) \nu^{\prime}\right]\right.} \\
+ & \left.\frac{2 \lambda^{\prime}}{r}+\frac{\mathrm{e}^{2 \lambda}}{r^{2}}\left\{1+\left\{-1-r\left(\nu^{\prime}-\lambda^{\prime}\right)\right\} \mathrm{e}^{-2 \lambda}\right\}\right] f^{\prime}(R) \\
+ & {\left[-\mathrm{e}^{-2 \nu+2 \lambda} f^{\prime}(R)-\left(\partial_{t} \partial_{r}-\left(\nu^{\prime} \partial_{t}-\dot{\lambda} \partial_{r}\right) f^{\prime}(R)\right.\right.} \\
V= & \left.\left.\frac{f(R)}{2}+\left[-\lambda^{\prime}-\frac{1}{r}\right) \partial_{r}\right)\right] f^{\prime}(R) \\
& \left.+\mathrm{e}^{-2 \lambda}\left\{\nu^{\prime \prime}+\left(\nu^{\prime}-\lambda^{\prime}\right) \nu^{\prime}+\frac{\nu^{\prime}-\lambda^{\prime}}{r}\right\}\right] f^{\prime}(R) \\
& +\frac{1}{2}\left[\mathrm{e}^{-2 \nu}\left\{\partial_{t}^{2}-(\dot{\lambda}-\dot{\lambda}) \partial_{t}\right\}\right. \\
& \left.-\mathrm{e}^{-2 \lambda}\left\{\partial_{r}^{2}+\left(\nu^{\prime}-\lambda^{\prime} \frac{4}{r}\right) \partial_{r}\right\}\right] f^{\prime}(R)
\end{aligned}
$$

Again, if we replace $t$ and $r$ with $\phi$ and $\chi$ in the righthand sides of Eqs. (4.6)-(4.9), we obtain $A, B, C$, and $V$ as functions of $\phi$ and $\chi$. Conversely if we assign $A, B, C$, and $V$, the model has a solution in the form of the spherically symmetric configuration (2.6) corresponding to arbitrary $\nu$ and $\lambda$.

In the case $\lambda=-\nu$ (or $g_{t t} g_{r r}=-1$ ) already discussed, we find

$$
\begin{aligned}
A= & {\left[-\frac{1}{2} \mathrm{e}^{2 \nu} \frac{d^{2}\left(\mathrm{e}^{-2 \nu}\right)}{d t^{2}}+\mathrm{e}^{2 \nu}\left\{\frac{1}{2} \frac{d^{2}\left(\mathrm{e}^{2 \nu}\right)}{d r^{2}}+\frac{1}{r} \frac{d\left(\mathrm{e}^{2 \nu}\right)}{d r}\right\}\right.} \\
& \left.-\frac{\mathrm{e}^{2 \nu}}{r^{2}}\left\{1+\left(-\mathrm{e}^{2 \nu}-r \frac{d\left(\mathrm{e}^{2 \nu}\right)}{d r}\right)\right\}\right] f^{\prime}(R) \\
& -\left[-\partial_{t}^{2}-\frac{\mathrm{e}^{2 \nu}}{2} \frac{d\left(\mathrm{e}^{-2 \nu}\right)}{d t} \partial_{t}+\left(\mathrm{e}^{2 \nu} \frac{d\left(\mathrm{e}^{2 \nu}\right)}{d r}+\frac{\mathrm{e}^{4 \nu}}{r}\right) \partial_{r}\right] f^{\prime}(R),
\end{aligned}
$$

$$
\begin{aligned}
B= & \frac{\mathrm{e}^{2 \nu}}{r} \frac{d\left(\mathrm{e}^{-2 \nu}\right)}{d t} f^{\prime}(R) \\
& -\left(\partial_{t} \partial_{r}-\frac{\mathrm{e}^{-2 \nu}}{2} \frac{d\left(\mathrm{e}^{2 \nu}\right)}{d r} \partial_{t}-\frac{\mathrm{e}^{2 \nu}}{2} \frac{d\left(\mathrm{e}^{-2 \nu}\right)}{d t} \partial_{r}\right) f^{\prime}(R),
\end{aligned}
$$

$$
\begin{aligned}
C= & {\left[\frac{\mathrm{e}^{-2 \nu}}{2} \frac{d^{2}\left(\mathrm{e}^{-2 \nu}\right)}{d t^{2}}-\frac{\mathrm{e}^{-2 \nu}}{2} \frac{d^{2}\left(\mathrm{e}^{2 \nu}\right)}{d r^{2}}-\frac{\mathrm{e}^{-2 \nu}}{2} \frac{d\left(\mathrm{e}^{2 \nu}\right)}{d r}\right.} \\
& \left.+\frac{\mathrm{e}^{-2 \nu}}{r^{2}}\left[1+\left\{-1-r \mathrm{e}^{-2 \nu} \frac{d\left(\mathrm{e}^{2 \nu}\right)}{d r}\right] \mathrm{e}^{2 \nu}\right\}\right] f^{\prime}(R) \\
& +\left[-\frac{\mathrm{e}^{-2 \nu}}{2} \frac{d\left(\mathrm{e}^{-2 \nu}\right)}{d t} \partial_{t}+\left(\partial_{r}^{2}+\left(\frac{\mathrm{e}^{-2 \nu}}{2} \frac{d\left(\mathrm{e}^{2 \nu}\right)}{d r}-\frac{1}{r}\right) \partial_{r}\right)\right] \\
& \times f^{\prime}(R), \\
V= & \frac{f(R)}{2}+\left[\frac{1}{2} \frac{d^{2}\left(\mathrm{e}^{-2 \nu}\right)}{d t^{2}}+\frac{1}{2} \frac{d^{2}\left(\mathrm{e}^{2 \nu}\right)}{d r^{2}}+\frac{1}{r} \frac{d\left(\mathrm{e}^{2 \nu}\right)}{d r}\right] f^{\prime}(R) \\
& +\frac{1}{2}\left[\mathrm{e}^{-2 \nu} \partial_{t}^{2}+\frac{d\left(\mathrm{e}^{-2 \nu}\right)}{d t} \partial_{t}-\mathrm{e}^{2 \nu} \partial_{r}^{2}-\left(\frac{d\left(\mathrm{e}^{2 \nu}\right)}{d r}+\frac{4}{r}\right) \partial_{r}\right] \\
& \times f^{\prime}(R) .
\end{aligned}
$$

Contrary to the Einstein gravity case (3.14), (3.15), and (3.16), it is now possible to avoid the ghost with a suitable choice of the Lagrangian density $f(R)$.

\section{A. Example 1}

With our previous ansatz (3.17) (with $\dot{M} \ll 1$ to ensure the adiabatic approximation), Eqs. (4.10)-(4.11) acquire the form

$$
\begin{aligned}
A= & -\left[\frac{\ddot{M}}{2 r}+\frac{\left(\frac{\dot{M}}{r}\right)^{2}}{2\left(1-\frac{M}{r}\right)}\right] f^{\prime}(R) \\
& -\left[-\partial_{t}^{2}-\frac{\frac{\dot{M}}{r}}{2\left(1-\frac{M}{r}\right)} \partial_{t}-\frac{1}{r}\left(1-\frac{2 M}{r}\right) \partial_{r}\right] f^{\prime}(R), \quad(4.14) \\
C= & \left(1-\frac{M}{r}\right)^{-2}\left[\frac{\ddot{M}}{2 r}+\frac{\left(\frac{\dot{M}}{r}\right)^{2}}{2\left(1-\frac{M}{r}\right)}\right] f^{\prime}(R) \\
& -\left[-\left(1-\frac{M}{r}\right)^{-3} \frac{\dot{M}}{2 r} \partial_{t}\right. \\
& \left.+\left(\partial_{r}^{2}-\frac{1}{2 r}\left(1-\frac{M}{r}\right)^{-1}\left(1-\frac{3 M}{r}\right) \partial_{r}\right)\right] f^{\prime}(R), \quad(4.15) \\
V= & \frac{f(R)}{2}+\left(1-\frac{M}{r}\right)^{-1}\left(\frac{\ddot{M}}{2 r}+\frac{\left(\frac{\dot{M}}{r}\right)^{2}}{2\left(1-\frac{M(t)}{r}\right)}\right) f^{\prime}(R) \\
& +\frac{1}{2}\left[\left(1-\frac{M}{r}\right)^{-1}\left\{\partial_{t}^{2}+\frac{\frac{\dot{M}}{r}}{1-\frac{M}{r}} \partial_{t}\right\}\right. \\
& \left.-\left(1-\frac{M}{r}\right)\left\{\partial_{r}^{2}+\frac{\frac{M}{r^{2}}+\frac{4}{1}}{1-\frac{M}{r}} \partial_{r}\right\}\right] f^{\prime}(R), \\
B= & \frac{1}{\kappa^{2}}\left(1-\frac{M}{r}\right)^{-1}\left(\frac{M^{\prime}}{r}-\partial_{t} \partial_{r}+\frac{M}{2 r^{2}} \partial_{t}+\frac{\dot{M}}{2 r} \partial_{r}\right) f^{\prime}(R) .
\end{aligned}
$$

Even in $f(R)$ gravity, it is difficult to avoid the ghost and we need to specify the model explicitly. 
As done for Eqs. (3.19)-(3.22), let us discuss the thermodynamics of the spacetime (3.17). Assuming again a quasistatic evolution $(M \ll 1)$ and treating the time $t$ as constant, the temperature is

$$
T=\frac{1}{8 \pi M(t)}
$$

whereas the Bekenstein-Hawking entropy reads

$\mathcal{S}=\frac{4 \pi[2 M(t)]^{2} f^{\prime}(R(r \rightarrow 2 M(t)))}{4}=\frac{f^{\prime}(+\infty)}{16 \pi T^{2}}$.

In order to obtain a nontrivial entropy, $f^{\prime}(+\infty)$ must be finite. The free energy is

$$
F=\frac{f^{\prime}(+\infty)}{16 \pi T},
$$

where we have chosen the integration constant so that $F$ vanishes in the limit $T \rightarrow \infty$ (or $M(t) \rightarrow 0$ ). The thermal energy is

$$
E=\frac{f^{\prime}(+\infty)}{8 \pi T}=f^{\prime}(+\infty) M(t),
$$

where a correction $f^{\prime}(+\infty)$ appears, as in the expression of $T$. This correction must be present, as one realizes by remembering that $f(R)$ gravity is equivalent to an $\omega=0$ Brans-Dicke theory [108] with the Brans-Dicke scalar $\phi_{\mathrm{BD}}=f^{\prime}(R)$ [109-111], which plays the role of the inverse of the effective gravitational coupling [108], $\phi_{\mathrm{BD}}=G_{\text {eff }}^{-1}$, and with a potential $V\left(\phi_{\mathrm{BD}}\right)$. Restoring Newton's constant $G$, the corresponding expressions in Brans-Dicke gravity contain $8 \pi G$ eff $=8 \pi / f^{\prime}(R)$.

\section{B. Example 2}

Moving on to the ansatz (3.24), we find

$$
\begin{aligned}
A= & -\frac{t_{0}}{t r^{2}}\left(1-\frac{r_{0}}{r}\right)\left(1-\frac{t_{0}}{t}\right) f^{\prime}(R) \\
& +\left[-\partial_{t}^{2}-\frac{1}{2 t} \partial_{t}+\left(1-\frac{r_{0}}{r}\right) \frac{t_{0}}{t r} \partial_{r}\right] f^{\prime}(R), \\
C= & \left(1-\frac{r_{0}}{r}\right)^{-1} \frac{1}{r^{2}}\left(\frac{t}{t_{0}}-1\right) f^{\prime}(R) \\
& -\left[-\left(1-\frac{r_{0}}{r}\right)^{-2} \frac{t}{2 t_{0}^{2}} \partial_{t}\right. \\
& \left.+\left(\partial_{r}^{2}+\frac{1}{2 r}\left(1-\frac{r_{0}}{r}\right)^{-1}\left(-2+\frac{3 r_{0}}{r}\right) \partial_{r}\right)\right] f^{\prime}(R),
\end{aligned}
$$

$$
\begin{gathered}
V=\frac{f(R)}{2}+\frac{1}{2}\left[\left(1-\frac{r_{0}}{r}\right)^{-1} \frac{t}{t_{0}}\left\{\partial_{t}^{2}+\frac{1}{t} \partial_{t}\right\}\right. \\
\left.+\left(1-\frac{r_{0}}{r}\right) \frac{t_{0}}{t}\left\{\partial_{r}^{2}-\frac{\frac{r_{0}}{r^{2}}+\frac{4}{r}}{1-\frac{r_{0}}{r}} \partial_{r}\right\}\right] f^{\prime}(R), \\
B=\frac{f^{\prime}(R)}{t r}-\left(\partial_{t} \partial_{r}-\frac{\frac{r_{0}}{r^{2}}}{2\left(1-\frac{r_{0}}{r}\right)} \partial_{t}-\frac{1}{2 t} \partial_{r}\right) f^{\prime}(R) .
\end{gathered}
$$

The Ricci scalar (3.28) yields

$$
\begin{aligned}
A= & -\frac{t_{0}}{t r^{2}}\left(1-\frac{r_{0}}{r}\right)\left(1-\frac{t_{0}}{t}\right) f^{\prime}(R)+\frac{4 t_{0}^{2}}{r^{4} t^{4}} f^{\prime \prime \prime}(R) \\
& +\left[\frac{3 t_{0}}{r^{2} t^{3}}-4\left(1-\frac{r_{0}}{r}\right) \frac{t_{0}^{2}}{t^{2} r^{4}}\left(1-\frac{t_{0}}{t}\right)\right] f^{\prime \prime}(R), \quad(4.26) \\
C= & \frac{t}{t_{0} r^{2}}\left(1-\frac{r_{0}}{r}\right)^{-1}\left(1-\frac{t_{0}}{t}\right) f^{\prime}(R)+\frac{16}{r^{6}}\left(1-\frac{t_{0}}{t}\right)^{2} f^{\prime \prime \prime}(R) \\
& -\left[-\left(1-\frac{r_{0}}{r}\right)^{-2} \frac{1}{t_{0} r^{2} t}\right. \\
& \left.+\frac{2}{r^{4}}\left(1-\frac{t_{0}}{t}\right)\left(1-\frac{r_{0}}{r}\right)^{-1}\left(8-\frac{9 r_{0}}{r}\right)\right] f^{\prime \prime}(R), \quad(4.27) \\
V= & \frac{f(R)}{2} \\
& +\frac{2 t_{0}}{r^{4} t^{3}}\left[\left(1-\frac{r_{0}}{r}\right)^{-1}+\frac{4 t_{0}}{r^{6} t}\left(1-\frac{r_{0}}{r}\right)\left(1-\frac{t_{0}}{t}\right)^{2}\right] f^{\prime \prime \prime}(R) \\
& -\left[-\frac{2}{r^{2} t^{2}}\left(1-\frac{r_{0}}{r}\right)^{-1}+\frac{t_{0}}{r^{4} t}\left(1-\frac{t_{0}}{t}\right)\left(28-\frac{8 r_{0}}{r}\right)\right] f^{\prime \prime}(R),
\end{aligned}
$$

$$
\begin{aligned}
B= & \frac{f^{\prime}(R)}{t r} \\
& -\left[-\frac{4 t_{0}}{r^{3} t^{2}}-\frac{r_{0} t_{0}}{r^{4} t^{2}}\left(1-\frac{r_{0}}{r}\right)^{-1}+\frac{2}{r^{3} t}\left(1-\frac{t_{0}}{t}\right)\right] f^{\prime \prime}(R) \\
& +\frac{8 t_{0}}{r^{3} t^{2}}\left(1-\frac{r_{0}}{r}\right) f^{\prime \prime \prime}(R) .
\end{aligned}
$$

In order to check whether the ghost can be avoided, let us examine the functions $A, B$, and $C$ in the regime of large $r$, in which one finds

$$
\begin{gathered}
A \sim-\frac{t_{0}}{t r^{2}}\left(1-\frac{t_{0}}{t}\right) f^{\prime}(R)+\frac{3 t_{0}}{r^{2} t^{3}} f^{\prime \prime}(R) \\
-\frac{4 t_{0}^{2}}{r^{4} t^{4}} f^{\prime \prime \prime}(R), \\
B \sim \frac{f^{\prime}(R)}{t r}-\frac{2}{r^{3} t}\left(1-\frac{t_{0}}{t}\right) f^{\prime \prime}(R)+\frac{8 t_{0}}{r^{3} t^{2}} f^{\prime \prime \prime}(R),
\end{gathered}
$$




$$
\begin{aligned}
C \sim & \frac{t}{t_{0} r^{2}}\left(1-\frac{t_{0}}{t}\right) f^{\prime}(R)+\frac{1}{t_{0} r^{2} t} f^{\prime \prime}(R) \\
& -\frac{16}{r^{6}}\left(1-\frac{t_{0}}{t}\right)^{2} f^{\prime \prime \prime}(R) .
\end{aligned}
$$

The popular power-law choice $([50,65,66,112-159]$, see $[160,161]$ for Solar System constraints on the parameter $n$ )

$$
f^{\prime}(R) \sim f_{0} R^{n}=f_{0}\left[\frac{2}{r^{2}}\left(1-\frac{t_{0}}{t}\right)\right]^{n}
$$

produces

$$
\begin{gathered}
A \sim-\frac{f_{0} n t_{0}}{r^{4} t^{3}}\left[\frac{2}{r^{2}}\left(1-\frac{t_{0}}{t}\right)\right]^{n-2}\left[-6+(2+4 n) \frac{t_{0}}{t}\right], \\
C \sim \frac{f_{0} n}{t_{0} r^{2} t}\left[\frac{2}{r^{2}}\left(1-\frac{t_{0}}{t}\right)\right]^{n-1},
\end{gathered}
$$

$B \sim \frac{f_{0}}{t r}\left[\frac{2}{r^{2}}\left(1-\frac{t_{0}}{t}\right)\right]^{n}-\frac{2 f_{0} n}{r^{3} t}\left(1-\frac{t_{0}}{t}\right)\left[\frac{2}{r^{2}}\left(1-\frac{t_{0}}{t}\right)\right]^{n-1}$.

The special case $n=1$ gives

$$
A \sim-\frac{3 f_{0} t_{0}}{r^{4} t^{3}}, \quad C \sim-\frac{f_{1}}{t_{0} r^{2} t}, \quad B \sim \mathcal{O}\left(r^{-4}\right)
$$

therefore, if $f_{0}<0$, both $A$ and $B$ are positive and

$$
A C-B^{2} \sim \frac{3 f_{0}^{2}}{r^{6} t^{4}},
$$

which tells us that, at least as long as $r$ is sufficiently large, there is no ghost. Thus, it seems possible to avoid ghosts in $f(R)$ gravity.

Again, we obtain the thermodynamics of this spacetime in the adiabatic approximation (assuming $|t| \gg t_{0}$ ). The temperature and the Bekenstein-Hawking entropy are

$$
\begin{gathered}
T=\frac{t_{0}}{4 \pi t r_{0}}, \\
\mathcal{S}=\frac{4 \pi f^{\prime}\left(R\left(r \rightarrow r_{0}\right)\right) r_{0}^{2}}{4}=\pi f^{\prime}\left(\frac{2}{r_{0}^{2}}\left(1-\frac{t_{0}}{t}\right)\right) r_{0}^{2},
\end{gathered}
$$

and they are both time dependent. Eliminating $r_{0}$ with Eq. (3.29) yields

$$
\mathcal{S}=\frac{t_{0}^{2}}{4 t^{2} T^{2}} f^{\prime}\left(\frac{32 \pi^{2} t^{2} T^{2}}{t_{0}^{2}}\left(1-\frac{t_{0}}{t}\right)\right) .
$$

For this explicit form of $f(R)$, the use of $d F / d T=-\mathcal{S}$ and $E=F+T S$ produces the nontrivial expressions of the free energy and the thermodynamical energy

$$
\begin{aligned}
F= & -\int \mathcal{S} d T=-\int \frac{t_{0}^{2} f^{\prime}\left(\frac{32 \pi^{2} t^{2} T^{2}}{t_{0}^{2}}\left(1-\frac{t_{0}}{t}\right)\right)}{4 t^{2} T^{2}} d T, \\
E= & F+T \mathcal{S}=\frac{t_{0}^{2} f^{\prime}\left(\frac{32 \pi^{2} t^{2} T^{2}}{t_{0}^{2}}\left(1-\frac{t_{0}}{t}\right)\right)}{4 t^{2} T} \\
& -\int \frac{t_{0}^{2} f^{\prime}\left(\frac{32 \pi^{2} t^{2} T^{2}}{t_{0}^{2}}\left(1-\frac{t_{0}}{t}\right)\right)}{4 t^{2} T^{2}} d T .
\end{aligned}
$$

By construction, these quantities satisfy the first law of thermodynamics $d E=T d \mathcal{S}=d Q$. For the model (4.33), we find

$$
\begin{aligned}
& F=-\frac{t_{0}^{2} f_{0}\left(\frac{32 \pi^{2} t^{2} T^{2}}{t_{0}^{2}}\left(1-\frac{t_{0}}{t}\right)\right)^{n}}{4(2 n-1) t^{2} T}, \\
& E=\frac{(n-1) t_{0}^{2} f_{0}\left(\frac{32 \pi^{2} t^{2} T^{2}}{t_{0}^{2}}\left(1-\frac{t_{0}}{t}\right)\right)^{n}}{2(2 n-1) t^{2} T} .
\end{aligned}
$$

When $t>t_{0}$, the thermodynamical energy $E$ is positive if $f_{0}>0$ and $n>1$ or $n<1 / 2$, or if $f_{0}<0$ and $1 / 2<n<1$. Moreover, when $t<t_{0}$ and $n$ is an integer, $E$ is positive in the following cases:

(i) $f_{0}>0, n$ is an even integer, and $n \geq 2$ or $n \leq 0$;

(ii) $f_{0}<0$ and $n=0$ (which corresponds to Einstein's gravity);

(iii) $f_{0}<0, n$ is an odd integer, and $n \geq 3$ or $n \leq-1$.

The cases with negative $E$ may be unphysical.

\section{Example 3}

For the example (3.39), we find

$$
\begin{aligned}
A= & \frac{\left(1-\frac{r_{0}}{r}\right)\left(1+\frac{t_{0}}{t}\right)\left(\frac{t}{t_{0}}-1+\frac{t r_{0}}{t_{0} r}\right) \frac{t r_{0}^{2}}{t_{0} r^{4}} f^{\prime}(R)}{\left(1+\frac{t r_{0}}{t_{0} r}\right)^{4}} \\
- & -\left[-\partial_{t}^{2}-\frac{\frac{r_{0}}{2 t_{0} r}}{1+\frac{t r_{0}}{t_{0} r}} \partial_{t}+\frac{\left(1-\frac{r_{0}}{r}\right)\left(1+\frac{t r_{0}}{t_{0} r}\right)}{r\left(1+\frac{t r_{0}}{t_{0}}\right)^{3}} \partial_{r}\right] f^{\prime}(R), \\
B= & \frac{\frac{r_{0}}{t_{0} r^{2}}}{1+\frac{t r_{0}}{t_{0} r}} f^{\prime}(R) \\
& -\left(\partial_{t} \partial_{r}-\frac{\left(1+\frac{t_{0}}{t}\right) \frac{t r_{0}}{2 t_{0} r^{2}}}{\left(1-\frac{r_{0}}{r}\right)\left(1+\frac{t r_{0}}{t_{0} r}\right)} \partial_{t}-\frac{\frac{r_{0}}{2 t_{0} r}}{1+\frac{t r_{0}}{t_{0} r}} \partial_{r}\right) f^{\prime}(R),
\end{aligned}
$$

$$
\begin{aligned}
& C=\left.-\frac{\left(1+\frac{t_{0}}{t}\right)\left(\frac{t}{t_{0}}-1+\frac{t r_{0}}{t_{0} r}\right) \frac{t r_{0}^{2}}{t_{0} r^{4}} f^{\prime}(R)+\left[-\frac{\left(1+\frac{t r_{0}}{t_{0} r}\right) \frac{r_{0}}{2 t_{0} r}}{\left(1-\frac{r_{0}}{r}\right)^{2}} \partial_{t}\right.}{t_{0} r}\right)^{2} \\
&\left.+\left(\partial_{r}^{2}+\left(\frac{\left(1+\frac{t_{0}}{t}\right) \frac{t r_{0}}{t_{0} r^{2}}}{\left(1-\frac{r_{0}}{r}\right)\left(1+\frac{t r_{0}}{t_{0} r}\right)}-\frac{1}{r}\right) \partial_{r}\right)\right] f^{\prime}(R), \quad(4.46)
\end{aligned}
$$




$$
\begin{aligned}
V= & \frac{f(R) f^{\prime}(R)}{2}\left[-\frac{\left(1+\frac{t_{0}}{t}\right) \frac{t r_{0}}{t_{0} r^{3}}}{\left(1+\frac{t r_{0}}{t_{0} r}\right)^{3}}+\frac{\left(1+\frac{t_{0}}{t}\right) \frac{t r_{0}}{t_{0} r^{3}}}{\left(1+\frac{t r_{0}}{t_{0} r}\right)^{2}}\right] \\
& +\frac{1}{2}\left[\frac{1+\frac{t r_{0}}{t_{0} r}}{1-\frac{r_{0}}{r}} \partial_{t}^{2}-\frac{\frac{r_{0}}{t_{0} r}}{1-\frac{r_{0}}{r}} \partial_{t}+\frac{1-\frac{r_{0}}{r}}{1+\frac{t r_{0}}{t_{0} r}} \partial_{r}^{2}\right. \\
& \left.-\left(\frac{\left(1+\frac{t_{0}}{t}\right) \frac{t r_{0}}{t_{0} r^{2}}}{\left(1+\frac{t r_{0}}{t_{0} r}\right)^{2}}+\frac{4}{r}\right) \partial_{r}\right] f^{\prime}(R) .
\end{aligned}
$$

The Hawking temperature and the entropy now read, in the adiabatic approximation $|t| \gg t_{0}$,

$$
\begin{gathered}
T=\frac{f^{\prime}\left(R\left(r \rightarrow r_{0}, t\right)\right)}{4 \pi r_{0}\left(1+\frac{t}{t_{0}}\right)}=\frac{f^{\prime}\left(\frac{\frac{2}{t_{0}^{2}}\left(1-\frac{t}{t_{0}}-\frac{t^{2}}{t_{0}^{2}}\right)}{\left(1+\frac{t}{t_{0}}\right)^{2}}\right)}{4 \pi r_{0}\left(1+\frac{t}{t_{0}}\right)}, \\
\mathcal{S}=\frac{4 \pi r_{0}^{2}}{4}=\frac{f^{\prime}\left(\frac{\frac{2}{r_{0}^{(}}\left(1-\frac{t}{t_{0}}-\frac{t^{2}}{t_{0}^{2}}\right)}{\left(1+\frac{t}{t_{0}}\right)^{2}}\right)^{2}}{16 \pi\left(1+\frac{t}{t_{0}}\right)^{2} T^{2}} .
\end{gathered}
$$

Nontrivial expressions of the free energy $F$ and the thermodynamical energy $E$ can be obtained as done with Eqs. (4.42) and (4.43). See Appendix B for further comments on $f(R)$ gravity with two scalar fields.

\section{SPHERICALLY SYMMETRIC, TIME-DEPENDENT GEOMETRY IN PURE $\boldsymbol{f}(\boldsymbol{R})$ GRAVITY}

It is difficult to avoid the ghost degree of freedom even in pure $f(R)$ gravity. In this section, in the framework of pure $f(R)$ gravity, we construct a model which admits a spherically symmetric and time-dependent solution.

Although the sought-for solution is time dependent, we begin with a model that gives a static solution. We choose $A=\alpha^{2}, B=C=0$, and $V=V_{0}=$ constant; then Eqs. (3.6)-(3.9) assume the form

$$
\begin{aligned}
& \frac{\mathrm{e}^{2(\nu-\lambda)}}{\kappa^{2}}\left(\frac{2 \lambda^{\prime}}{r}+\frac{\mathrm{e}^{2 \lambda}-1}{r^{2}}\right)=-\mathrm{e}^{2 \nu}\left(\frac{\alpha^{2}}{2} \mathrm{e}^{-2 \nu}+V_{0}\right), \\
& \frac{1}{\kappa^{2}}\left(\frac{2 \nu^{\prime}}{r}-\frac{\mathrm{e}^{2 \lambda}-1}{r^{2}}\right)=-\mathrm{e}^{2 \lambda}\left(\frac{\alpha^{2}}{2} \mathrm{e}^{-2 \nu}-V_{0}\right), \\
& \frac{1}{\kappa^{2}}\left[\mathrm{e}^{-2 \lambda}\left(r\left(\nu^{\prime}-\lambda^{\prime}\right)+r^{2} \nu^{\prime \prime}+r^{2}\left(\nu^{\prime}-\lambda^{\prime}\right) \nu^{\prime}\right)\right] \\
& =-r^{2}\left(\frac{\alpha^{2}}{2} \mathrm{e}^{-2 \nu}-V_{0}\right) .
\end{aligned}
$$

Equations (5.1) and (5.2) give

$$
\lambda^{\prime}=-\frac{\left(\mathrm{e}^{2 \lambda}-1\right)}{2 r}-\frac{\kappa^{2} r \mathrm{e}^{2 \lambda}}{2}\left(\frac{\alpha^{2} \mathrm{e}^{-2 \nu}}{2}+V_{0}\right),
$$

$$
\nu^{\prime}=\frac{\mathrm{e}^{2 \lambda}-1}{2 r}-\frac{\kappa^{2} r \mathrm{e}^{2 \lambda}}{2}\left(\frac{\alpha^{2}}{2} \mathrm{e}^{-2 \nu}-V_{0}\right) .
$$

By using Eqs. (5.4) and (5.5), one checks that Eq. (5.3) is automatically satisfied. Therefore, we can choose (5.4) and (5.5) as our two independent equations. By solving them with physically realistic boundary conditions, one obtains a spherically symmetric and static solution $\nu=\nu_{\mathrm{ss}}(r)$ and $\lambda=\lambda_{\mathrm{ss}}(r)$.

Now the action has the form

$$
S_{\phi}=\int d^{4} x \sqrt{-g}\left(\frac{R}{2 \kappa^{2}}-\frac{\alpha^{2}}{2} \partial_{\mu} \phi \partial^{\mu} \phi-V_{0}\right)
$$

describing GR with a single, canonical, scalar field. Let us examine the relation between the model (5.6) and $f(R)$ gravity. Under the conformal transformation $g_{\mu \nu} \rightarrow \tilde{g}_{\mu \nu}=$ $\mathrm{e}^{\rho} g_{\mu \nu}$, the Ricci scalar transforms according to [1]

$$
R \rightarrow \tilde{R}=\left(R-3 \square \rho-\frac{3}{2} \partial^{\mu} \rho \partial_{\mu} \rho\right) \mathrm{e}^{-\rho}
$$

and the action (5.6) is mapped into

$$
\begin{aligned}
S_{\phi}= & \int d^{4} x \sqrt{-g} \mathrm{e}^{2 \rho}\left[\frac{1}{2 \kappa^{2}}\left(R-3 \square \rho-\frac{3}{2} \partial^{\mu} \rho \partial_{\mu} \rho\right) \mathrm{e}^{-\rho}\right. \\
& \left.-\frac{\alpha^{2}}{2} \mathrm{e}^{-\rho} \partial_{\mu} \phi \partial^{\mu} \phi-V_{0}\right] .
\end{aligned}
$$

The choice $\rho=\phi / \sqrt{3}$ yields

$$
S_{\phi}=\int d^{4} x \sqrt{-g}\left(\frac{1}{2 \kappa^{2}} \mathrm{e}^{\frac{\phi}{\sqrt{3}}} R-\mathrm{e}^{\frac{2 \phi}{\sqrt{3}}} \tilde{V}_{0}\right)
$$

and the variation of the action (5.9) with respect to $\phi$ yields the algebraic equation

$$
\frac{1}{2 \kappa^{2}} \mathrm{e}^{\frac{\phi}{\sqrt{3}}} R-2 \mathrm{e}^{\frac{2 \phi}{\sqrt{3}}} V_{0}=0
$$

for $\mathrm{e}^{\phi / \sqrt{3}}$, which has the unique root

$$
\mathrm{e}^{\phi / \sqrt{3}}=\frac{R}{4 \kappa^{2} V_{0}} .
$$

By substituting Eq. (5.11) into the action (5.9), one obtains the $R^{2}$ gravity model

$$
S_{\phi}=-\frac{1}{8 \kappa^{4} V_{0}} \int d^{4} x \sqrt{-g} R^{2} .
$$

Due to the conformal transformation $g_{\mu \nu} \rightarrow \tilde{g}_{\mu \nu} \equiv \mathrm{e}^{\rho} g_{\mu \nu}=$ $\mathrm{e}^{\phi / \sqrt{3}} g_{\mu \nu}$, the spacetime metric that solves Eqs. (5.4) and (5.5) differs from $g_{\mu \nu}$ by the conformal factor $\mathrm{e}^{-\phi / \sqrt{3}}=\mathrm{e}^{-t / \sqrt{3}}$, or 


$$
\begin{aligned}
d s^{2}= & \mathrm{e}^{-\frac{t}{\sqrt{3}}}\left[-\mathrm{e}^{2 \nu_{\mathrm{ss}}(r)} d t^{2}+\mathrm{e}^{2 \lambda_{\mathrm{ss}}(r)} d r^{2}\right. \\
& \left.+r^{2}\left(d \vartheta^{2}+\sin ^{2} \vartheta d \varphi^{2}\right)\right],
\end{aligned}
$$

a time-dependent line element.

Let us discuss now the solutions (5.4) and (5.5) in more detail. Define $X_{ \pm}=\mathrm{e}^{\nu \pm \lambda}$ and rewrite these equations as

$$
\begin{aligned}
& X_{+}^{\prime}=-\kappa^{2} r X_{+}^{2} X_{-}^{-1}\left(\frac{\alpha^{2}}{2} X_{+}^{-1} X_{-}^{-1}+V_{0}\right), \\
& X_{-}^{\prime}=\frac{X_{-}\left(X_{+} X_{-}^{-1}-1\right)}{2 r} .
\end{aligned}
$$

There exists a nontrivial fixed point when $V_{0}<0$ as $X_{+}=X_{-}=\sqrt{-\frac{\alpha^{2}}{2 V_{0}}}$. Therefore, if $-\frac{\alpha^{2}}{2 V_{0}}=1$, there is an asymptotically flat spacetime. In the following, we assume that $V_{0}=-\alpha^{2} / 2$ and we rewrite Eq. (5.14) as

$$
\begin{aligned}
& X_{+}^{\prime}=-\frac{\kappa^{2} \alpha^{2}}{2} r X_{+}^{2} X_{-}^{-1}\left(X_{+}^{-1} X_{-}^{-1}-1\right), \\
& X_{-}^{\prime}=\frac{X_{-}\left(X_{+} X_{-}^{-1}-1\right)}{2 r} .
\end{aligned}
$$

Note also that $X_{+}^{\prime}=0$ when $X_{+} X_{-}=1$ and $X_{-}^{\prime}=0$ when $X_{+}=X_{-}$. More precisely, the situation is summarized as follows:

(1) When both $X_{+}$and $X_{-}>0$,

(a) if $X_{-}>X_{+}^{-1}$, then $X_{+}^{\prime}>0$;

(b) if $X_{-}<X_{+}^{-1}$, then $X_{+}^{\prime}<0$; and

(a) if $X_{-}>X_{+}$, then $X_{-}^{\prime}<0$;

(b) if $X_{-}<X_{+}$, then $X_{-}^{\prime}>0$.

(2) When $X_{+}<0$ and $X_{-}>0$, then $X_{+}^{\prime}>0$ and $X_{-}^{\prime}<0$.

(3) When $X_{+}, X_{-}<0$,

(a) if $X_{-}>X_{+}^{-1}$, then $X_{+}^{\prime}>0$;

(b) if $X_{-}<X_{+}^{-1}$, then $X_{+}^{\prime}<0$.

and

(a) if $X_{-}>X_{+}$, then $X_{-}^{\prime}<0$;

(b) if $X_{-}<X_{+}$, then $X_{-}^{\prime}>0$.

(4) When $X_{+}>0$, we have $X_{-}<0, X_{+}^{\prime}<0$, and $X_{-}^{\prime}>0$.

We also note that $X_{+}^{\prime} \rightarrow 0$ as $X_{+} \rightarrow 0$. Furthermore, when $X_{-} \rightarrow 0$, then $X_{+}^{\prime} \rightarrow-\infty\left(X_{+}>0\right)$ and $X_{+}^{\prime} \rightarrow$ $+\infty\left(X_{+}<0\right)$. In the Schwarzschild-like solution with $g_{t t} g_{r r}=-1, X_{+}=1$ and $X_{-}$vanishes at horizon. Therefore, the solution with $g_{t t} g_{r r}=-1$ does not exist in the model (5.14).

We now perturb the fixed points $X_{ \pm}=1$ as described by $X_{ \pm}=1+\delta X_{ \pm}$. Equation (5.14) yields

$$
\begin{gathered}
\delta X_{+}{ }^{\prime}=\frac{\kappa^{2} \alpha^{2} r}{2}\left(\delta X_{+}+\delta X_{-}\right) \\
\delta X_{-}^{\prime}=\frac{1}{2 r}\left(\delta X_{+}-\delta X_{-}\right) .
\end{gathered}
$$

The eigenvalues $\lambda$ of the matrix

$$
\hat{M} \equiv\left(\begin{array}{cc}
\frac{\kappa^{2} \alpha^{2} r}{2} & \frac{\kappa^{2} \alpha^{2} r}{2} \\
\frac{1}{2 r} & -\frac{1}{2 r}
\end{array}\right)
$$

are the roots of the secular equation

$$
\lambda^{2}-\left(\frac{\kappa^{2} \alpha^{2} r}{2}-\frac{1}{2 r}\right)-\frac{\kappa^{2} \alpha^{2}}{4}=\left(\lambda-\frac{\kappa^{2} \alpha^{2} r}{2}\right)\left(\lambda+\frac{1}{2 r}\right)=0,
$$

hence both eigenvalues $\kappa^{2} \alpha^{2} r / 2$ and $-1 /(2 r)$ are negative and the equilibrium point $X_{ \pm}=1$ is a saddle point. The negative eigenvalue $-1 /(2 r)$ corresponds to the spacetime where $r$ becomes larger, $X_{ \pm} \rightarrow 1$; therefore, this geometry is asymptotically flat.

There is also a line of (nonisolated) fixed points at $X_{-} \rightarrow \infty$. To see this, we rewrite Eq. (5.15) as

$$
\begin{aligned}
X_{+}^{\prime} & =-\frac{\kappa^{2} \alpha^{2}}{2} r X_{+}^{2} X_{-}^{-1}\left(X_{+}^{-1} X_{-}^{-1}-1\right), \\
\left(X_{-}^{-1}\right)^{\prime} & =-\frac{X_{-}^{-1}\left(X_{+} X_{-}^{-1}-1\right)}{2 r} .
\end{aligned}
$$

Then, at $X_{-}^{-1}=0$ we find $X_{+}^{\prime}=\left(X_{-}^{-1}\right)^{\prime}=0$. In order to investigate the stability, we consider the perturbation $\delta X_{-}^{-1}$ of $X_{-}^{-1}$ by fixing $X_{+}$. The second equation in (5.20) reduces to

$$
\left(\delta X_{-}^{-1}\right)^{\prime} \sim \frac{\delta X_{-}^{-1}}{2 r} .
$$

The eigenvalue $1 /(2 r)$ is always positive.

Then there are several types of solutions. By fine-tuning the initial condition, one could find a trajectory which begins near $X_{-} \rightarrow+\infty$ and approaches the fixed point $X_{ \pm}=1$. There could also be trajectories beginning near $X_{-} \rightarrow+\infty$, crossing the line $X_{+}=X_{-}$, and approaching to $X_{+} \rightarrow+\infty$. The details are not particularly illuminating and will not be reported; however, the main point is that the existence of time-dependent and spherically symmetric solutions in pure $f(R)$ gravity is determined.

\section{CONCLUSIONS}

Many of our examples are plagued by the presence of a ghost, a phantom scalar field with negative kinetic energy. Such phantom fields suffer from instability and should not exist; however, from time to time cosmological observations argue in favor of a phantom equation of state of the cosmic quintessence fluid [162-166]. Should such claims persist, one would be forced to take the phantom phenomenology more seriously, perhaps not as signaling a true phantom field, but as a phantomlike phenomenon arising in 
a theory that is fundamentally ghost-free (this happens occasionally in modified gravity, for example in $f(R)$ gravity, whose gravitational sector is ghost-free [109111]). In this case, a model with a phantom field would be a mimicker of a more viable theory and the black hole solutions described in the previous sections would then become more interesting. These solutions include a static apparent horizon in a time-dependent black hole geometry, in which the black hole accretes scalar fluid and changes its temperature, while keeping its horizon unchanged. The first of our three ansatzes produces genuine time-dependent black holes with apparent horizons scaling in time with the Misner-Sharp-Hernandez mass (this horizon will look the same to all observers associated with a spherically symmetric foliation [14], but not to those moving with respect to the former in such a way that this symmetry is broken $[9,10]$, for example by a Lorentz boost along a nonradial direction).

Thus far, solving directly the field equations of various theories has not produced many physically reasonable solutions describing dynamical black holes; naked singularities and wormholes are much more common [8,73-86]. Another problem is that some of these analytical solutions are cumbersome when expressed in terms of the areal radius (e.g., $[20,21,65,67])$ and often the apparent horizons cannot be located analytically, or the analytical expressions providing them are not explicit [8]. Overall, designing time-dependent black holes is difficult and we reverse engineered the coupling functions of theories of gravity that admit prescribed apparent horizons as their solutions. Simpler analytical dynamical black holes will be searched for in future work.

\section{ACKNOWLEDGMENTS}

We thank a referee for useful comments leading to an improved presentation. This work was supported by MINECO (Spain), Projects No. PID2019-104397 GB-I00 and No. PHAROS COST Action (CA16214) (S. D. O.), by JSPS Grant-in-Aid for Scientific Research (C) No. 18K03615 (S. N.), and by the Natural Sciences \& Engineering Research Council of Canada, Grant No. 2016-03803 (V. F.). The work by S. D. O. was partially supported by the Ministry of Science and High Education of Russia, Project No. FEWF-2020-003.

\section{APPENDIX A}

When the metric can be regarded as static, or its time dependence can be neglected, we consider the line element

$$
\begin{aligned}
d s^{2}= & -P(r)\left(r-r_{0}\right) d t^{2}+\frac{d r^{2}}{P(r)\left(r-r_{0}\right)} \\
& +r^{2}\left(d \vartheta^{2}+\sin ^{2} \vartheta d \varphi^{2}\right) .
\end{aligned}
$$

We assume that $P(r)$ is positive everywhere and is a sufficiently smooth function of $r$ near the horizon $r=r_{0}$, and that it can be approximated by a constant, $P(r) \sim P\left(r_{0}\right)$. We then introduce a new coordinate $\rho$ by

$$
d \rho=\frac{d r}{\sqrt{P\left(r_{0}\right)\left(r-r_{0}\right)}},
$$

that is,

$$
\rho=2 \sqrt{\frac{r-r_{0}}{P\left(r_{0}\right)}} .
$$

We also Wick-rotate the time coordinate as $t \rightarrow i \tau$. Then, we obtain the Euclidean metric

$d s^{2}=\frac{P\left(r_{0}\right)^{2}}{4} \rho^{2} d \tau^{2}+d \rho^{2}+r(\rho)^{2}\left(d \vartheta^{2}+\sin ^{2} \vartheta d \varphi^{2}\right)$.

In order to avoid the conical singularity at $\rho=0$, we need to impose the periodicity on $\tau$,

$$
\frac{P\left(r_{0}\right)}{2} \tau \sim \frac{P\left(r_{0}\right)}{2} \tau+2 \pi
$$

For the finite temperature formalism in the path integral, the periodicity $\frac{4 \pi}{P\left(r_{0}\right)}$ corresponds to the inverse of the temperature

$$
T=\frac{P\left(r_{0}\right)}{4 \pi} .
$$

In the case of the metric given by (3.24), we find

$$
P\left(r_{0}\right)=\frac{t_{0}}{t r_{0}}
$$

and we obtain the expression (3.29). $t$ and $T$ are the Kodama time and Kodama temperature, respectively.

\section{APPENDIX B}

The action for Einstein gravity with a scalar field doublet is

$$
\begin{aligned}
S_{(\phi \chi)}= & \int d^{4} x \sqrt{-g}\left\{\frac{R}{2 \kappa^{2}}-\frac{1}{2} A(\phi, \chi) \partial_{\mu} \phi \partial^{\mu} \phi\right. \\
& -B(\phi, \chi) \partial_{\mu} \phi \partial^{\mu} \chi \\
& \left.-\frac{1}{2} C(\phi, \chi) \partial_{\mu} \chi \partial^{\mu} \chi-V(\phi, \chi)\right\} .
\end{aligned}
$$

In the static case where $B=0$, the action (B1) reduces to

$$
\begin{aligned}
S_{(\phi \chi)}= & \int d^{4} x \sqrt{-g}\left\{\frac{R}{2 \kappa^{2}}-\frac{1}{2} A(\chi) \partial_{\mu} \phi \partial^{\mu} \phi\right. \\
& \left.-\frac{1}{2} C(\chi) \partial_{\mu} \chi \partial^{\mu} \chi-V(\chi)\right\} .
\end{aligned}
$$


We now redefine the scalar field $\chi$ as

$$
\tilde{\chi}=\int d \chi \sqrt{|C(\chi)|}
$$

and we rewrite the action (B2) as

$$
\begin{aligned}
& S_{(\phi \chi)}= \int d^{4} x \sqrt{-g}\left\{\frac{R}{2 \kappa^{2}}-\frac{1}{2} \tilde{A}(\tilde{\chi}) \partial_{\mu} \phi \partial^{\mu} \phi\right. \\
&\left.-\frac{1}{2} \operatorname{sgn}(C) \partial_{\mu} \tilde{\chi} \partial^{\mu} \tilde{\chi}-\tilde{V}(\tilde{\chi})\right\}, \\
& \tilde{A}(\tilde{\chi}) \equiv A(\chi(\tilde{\chi})), \quad \tilde{V}(\tilde{\chi}) \equiv V(\chi(\tilde{\chi})),
\end{aligned}
$$

where

$$
\operatorname{sgn}(C) \equiv \begin{cases}+1 & \text { when } C>0 \\ -1 & \text { when } C<0\end{cases}
$$

We now consider the relation between the model (B2) and $F(R)$ gravity, or models similar to $F(R)$ gravity. For this purpose, assume that $C>0$, that is, $\operatorname{sgn}(C)=1$. Under the scale transformation $g_{\mu \nu} \rightarrow \mathrm{e}^{\rho} g_{\mu \nu}$, the Ricci scalar transforms as

$$
R \rightarrow\left(R-3 \square \rho-\frac{3}{2} \partial^{\mu} \rho \partial_{\mu} \rho\right) \mathrm{e}^{-\rho}
$$

and the action (B2) with $\operatorname{sgn}(C)=1$ transforms as

$$
\begin{aligned}
S_{(\phi \chi)} & =\int d^{4} x \sqrt{-g} \mathrm{e}^{2 \rho}\left\{\frac{1}{2 \kappa^{2}}\left(R-3 \square \rho-\frac{3}{2} \partial^{\mu} \rho \partial_{\mu} \rho\right) \mathrm{e}^{-\rho}\right. \\
& \left.-\frac{1}{2} \tilde{A}(\tilde{\chi}) \mathrm{e}^{-\rho} \partial_{\mu} \phi \partial^{\mu} \phi-\frac{1}{2} \mathrm{e}^{-\rho} \partial_{\mu} \tilde{\chi} \partial^{\mu} \tilde{\chi}-\tilde{V}(\tilde{\chi})\right\} .
\end{aligned}
$$

Then, the choice $\rho=\tilde{\chi} / \sqrt{3}$ yields

$$
\begin{aligned}
S_{(\phi \chi)}= & \int d^{4} x \sqrt{-g}\left\{\frac{1}{2 \kappa^{2}} \mathrm{e}^{\frac{\tilde{\chi}}{\sqrt{3}}} R-\frac{1}{2} \tilde{A}(\tilde{\chi}) \mathrm{e}^{\frac{\tilde{\chi}}{\sqrt{3}}} \partial_{\mu} \phi \partial^{\mu} \phi\right. \\
& -\mathrm{e}^{\left.\frac{2 \tilde{\chi}}{\sqrt{3}} \tilde{V}(\tilde{\chi})\right\} .}
\end{aligned}
$$

By varying with respect to $\tilde{\chi}$, one obtains the algebraic equation

$$
\begin{aligned}
0= & \frac{1}{\sqrt{3}}\left\{\frac{1}{2 \kappa^{2}} \mathrm{e}^{\frac{\tilde{x}}{\sqrt{3}}} R-\frac{1}{2} \tilde{A}(\tilde{\chi}) \mathrm{e}^{\frac{\tilde{x}}{\sqrt{3}}} \partial_{\mu} \phi \partial^{\mu} \phi-2 \mathrm{e}^{\frac{2 \tilde{\chi}}{\sqrt{3}}} \tilde{V}(\tilde{\chi})\right\} \\
& -\frac{1}{2} \tilde{A}^{\prime}(\tilde{\chi}) \mathrm{e}^{\frac{\tilde{\chi}}{\sqrt{3}}} \partial_{\mu} \phi \partial^{\mu} \phi-\mathrm{e}^{\frac{2 \tilde{x}}{\sqrt{3}}} \tilde{V}^{\prime}(\tilde{\chi}),
\end{aligned}
$$

which can, in principle, be solved with respect to $\tilde{\chi}$ as $\tilde{\chi}=\tilde{\chi}\left(R, \partial_{\mu} \phi \partial^{\mu} \phi\right)$. Then by substituting the expression of $\tilde{\chi}\left(R, \partial_{\mu} \phi \partial^{\mu} \phi\right)$ into the action (B8), we obtain an action which is, in a sense, similar to the action of $F(R)$ gravity,

$$
S_{(\phi \chi)}=\int d^{4} x \sqrt{-g} F\left(R, \partial_{\mu} \phi \partial^{\mu} \phi\right)
$$

where

$$
\begin{aligned}
F\left(R, \partial_{\mu} \phi \partial^{\mu} \phi\right) \equiv & \frac{1}{2 \kappa^{2}} \mathrm{e}^{\frac{\tilde{\chi}\left(R, \partial_{\mu} \phi \partial^{\mu} \phi\right)}{\sqrt{3}} R} \\
& -\frac{1}{2} \tilde{A}\left(\tilde{\chi}\left(R, \partial_{\mu} \phi \partial^{\mu} \phi\right)\right) \mathrm{e}^{\frac{\tilde{\chi}\left(R, \partial_{\mu} \phi \partial^{\mu} \phi\right)}{\sqrt{3}}} \partial_{\mu} \phi \partial^{\mu} \phi \\
& -\mathrm{e}^{\frac{2 \tilde{\chi}\left(R, \partial_{\mu} \phi \partial^{\mu} \phi\right)}{\sqrt{3}}} \tilde{V}\left(\tilde{\chi}\left(R, \partial_{\mu} \phi \partial^{\mu} \phi\right)\right) .
\end{aligned}
$$

Therefore, even in the static case, Einstein's gravity with two scalar fields cannot be rewritten in a simple $F(R)$ gravity form, but we obtain a rather complicated model instead.

In the time-dependent case it might be possible, in principle, to rewrite the model in a way similar to the form of $F(R)$ gravity, although the situation is not simple since the model obtained in this way assumes very complicated forms.

The physical nature of analytic solutions of the field equations may change radically when one goes from static to time-dependent solutions. In GR with a single scalar field, static black holes are Schwarzschild, or else the Fisher solution, which describes a naked singularity, is obtained for a single, static, asymptotically flat scalar field. However, when the geometry and the free scalar field are allowed to be time dependent, one obtains the HusainMartinez-Nuñez solution, which describes a cosmological black hole for part of its history [41]. Similar conclusions are reached by introducing an exponential potential for the scalar field, which produces the Fonarev solution [82].

When one contemplates time-dependent, asymptotically FLRW solutions of the Einstein equations with a fluid, one finds the McVittie metric [19]. The latter cannot be generated as a scalar field solution of the Einstein equations, or as a solution of scalar-tensor gravity. However, it is an exact solution of cuscuton theory, a special case of Hořava-Lifschitz gravity [39], which is the only form of $k$-essence that admits McVittie solutions [39]. The McVittie spacetime is also an solution of shape dynamics [167], and of $f(T)$ gravity (where $T$ is the torsion scalar [168]). $f(R)$ gravity with a single scalar is essentially equivalent to GR with two scalars and, while one does not expect much difference from the case of GR with a single scalar in the static case, one expects the time-dependent, asymptotically FLRW situation to produce more analytic solutions which contain dynamical black hole apparent horizons for part of the spacetime history, as in the case of the Husain-MartinezNuñez [41] and Fonarev [82] solutions. 
[1] R. M. Wald, General Relativity (Chicago University Press, Chicago, 1984).

[2] E. Poisson, A Relativist's Toolkit: The Mathematics of Black-Hole Mechanics (Cambridge University Press, Cambridge, England, 2004).

[3] R. M. Wald, Living Rev. Relativity 4, 6 (2001).

[4] A. B. Nielsen, Gen. Relativ. Gravit. 41, 1539 (2009).

[5] A. B. Nielsen, Classical Quantum Gravity 27, 245016 (2010).

[6] I. Booth, Can. J. Phys. 83, 1073 (2005).

[7] V. Faraoni, Universe 4, 109 (2018).

[8] V. Faraoni, Cosmological and Black Hole Apparent Horizons (Springer, New York, 2015).

[9] R. M. Wald and V. Iyer, Phys. Rev. D 44, R3719 (1991).

[10] E. Schnetter and B. Krishnan, Phys. Rev. D 73, 021502 (2006).

[11] B. P. Abbott et al. (LIGO Scientific and Virgo Collaborations), Phys. Rev. Lett. 116, 061102 (2016).

[12] B. P. Abbott et al. (LIGO Scientific and Virgo Collaborations), Phys. Rev. Lett. 116, 241103 (2016).

[13] B. P. Abbott et al. (LIGO Scientific and VIRGO Collaborations), Phys. Rev. Lett. 118, 221101 (2017); 121 (2018) 129901(E).

[14] V. Faraoni, G. F. R. Ellis, J. T. Firouzjaee, A. Helou, and I. Musco, Phys. Rev. D 95, 024008 (2017).

[15] C. W. Misner and D. H. Sharp, Phys. Rev. 136, B571 (1964).

[16] W. C. Hernandez and C. W. Misner, Astrophys. J. 143, 452 (1966).

[17] S. Hawking, J. Math. Phys. (N.Y.) 9, 598 (1968).

[18] S. A. Hayward, Phys. Rev. D 49, 831 (1994).

[19] G. C. McVittie, Mon. Not. R. Astron. Soc. 93, 325 (1933).

[20] V. Faraoni and A. Jacques, Phys. Rev. D 76, 063510 (2007).

[21] C. Gao, X. Chen, V. Faraoni, and Y. G. Shen, Phys. Rev. D 78, 024008 (2008).

[22] B. C. Nolan, J. Math. Phys. (N.Y.) 34, 178 (1993).

[23] B. C. Nolan, Phys. Rev. D 58, 064006 (1998).

[24] B. C. Nolan, Classical Quantum Gravity 16, 1227 (1999).

[25] B. C. Nolan, Classical Quantum Gravity 16, 3183 (1999).

[26] M. Ferraris, M. Francaviglia, and A. Spallicci, Nuovo Cimento B 111, 1031 (1996).

[27] N. Kaloper, M. Kleban, and D. Martin, Phys. Rev. D 81, 104044 (2010).

[28] K. Lake and M. Abdelqader, Phys. Rev. D 84, 044045 (2011).

[29] P. Landry, M. Abdelqader, and K. Lake, Phys. Rev. D 86, 084002 (2012).

[30] R. Nandra, A. N. Lasenby, and M. P. Hobson, Mon. Not. R. Astron. Soc. 422, 2931 (2012).

[31] R. Nandra, A. N. Lasenby, and M. P. Hobson, Mon. Not. R. Astron. Soc. 422, 2945 (2012).

[32] M. Carrera and D. Giulini, Phys. Rev. D 81, 043521 (2010).

[33] A. Maciel, D. C. Guariento, and C. Molina, Phys. Rev. D 91, 084043 (2015).

[34] A. M. da Silva, M. Fontanini, and D. C. Guariento, Phys. Rev. D 87, 064030 (2013).

[35] M. Le Delliou, J. P. Mimoso, F. C. Mena, M. Fontanini, D. C. Guariento, and E. Abdalla, Phys. Rev. D 88, 027301 (2013).
[36] V. Faraoni, A. F. Z. Moreno, and A. Prain, Phys. Rev. D 89, 103514 (2014).

[37] V. Faraoni, A. F. Z. Moreno, and R. Nandra, Phys. Rev. D 85, 083526 (2012).

[38] D. Gregoris, Y. C. Ong, and B. Wang, Eur. Phys. J. C 80, 159 (2020).

[39] E. Abdalla, N. Afshordi, M. Fontanini, D. C. Guariento, and E. Papantonopoulos, Phys. Rev. D 89, 104018 (2014).

[40] N. Afshordi, M. Fontanini, and D. C. Guariento, Phys. Rev. D 90, 084012 (2014).

[41] V. Husain, E. A. Martinez, and D. Nunez, Phys. Rev. D 50, 3783 (1994)

[42] S. N. G. Thakurta, Indian J. Phys. 55B, 304 (1981).

[43] V. Faraoni, C. Gao, X. Chen, and Y. G. Shen, Phys. Lett. B 671, 7 (2009).

[44] J. Sultana and C. C. Dyer, Gen. Relativ. Gravit. 37, 1347 (2005).

[45] M. L. McClure and C. C. Dyer, Classical Quantum Gravity 23, 1971 (2006).

[46] M. L. McClure and C. C. Dyer, Gen. Relativ. Gravit. 38, 1347 (2006).

[47] M. L. McClure, arXiv:0709.3288.

[48] M. L. McClure, K. Anderson, and K. Bardahl, Phys. Rev. D 77, 104008 (2008).

[49] C. J. Gao and S. N. Zhang, Phys. Lett. B 595, 28 (2004).

[50] S. Nojiri, S. D. Odintsov, and V. K. Oikonomou, Classical Quantum Gravity 34, 245012 (2017).

[51] T. K. Finch and J. Lindesay, arXiv:1110.6928.

[52] J. Lindesay, Foundations of Quantum Gravity (Cambridge University Press, Cambridge, England, 2013).

[53] J. Lindesay and P. Sheldon, Classical Quantum Gravity 27, 215015 (2010).

[54] M. Nozawa and H. Maeda, Classical Quantum Gravity 25, 055009 (2008).

[55] M. Pielahn, G. Kunstatter, and A. B. Nielsen, Phys. Rev. D 84, 104008 (2011).

[56] M. G. Rodrigues and V. T. Zanchin, Classical Quantum Gravity 32, 115004 (2015).

[57] H. Saida, T. Harada, and H. Maeda, Classical Quantum Gravity 24, 4711 (2007).

[58] N. Sakai and J. D. Barrow, Classical Quantum Gravity 18, 4717 (2001).

[59] R. Sussman, Gen. Relativ. Gravit. 17, 251 (1985).

[60] V. Faraoni, Phys. Rev. D 76, 104042 (2007).

[61] V. Faraoni, Phys. Rev. D 80, 044013 (2009).

[62] V. Faraoni, Phys. Rev. D 81, 044002 (2010).

[63] A. B. Nielsen and V. Faraoni, Classical Quantum Gravity 28, 175008 (2011).

[64] D. C. Guariento, A. Maciel, M. M. C. Mello, and V. T. Zanchin, Phys. Rev. D 100, 104050 (2019).

[65] T. Clifton, Classical Quantum Gravity 23, 7445 (2006).

[66] V. Faraoni, Classical Quantum Gravity 26, 195013 (2009).

[67] T. Clifton, D. F. Mota, and J. D. Barrow, Mon. Not. R. Astron. Soc. 358, 601 (2005).

[68] V. Faraoni, V. Vitagliano, T. P. Sotiriou, and S. Liberati, Phys. Rev. D 86, 064040 (2012).

[69] S. W. Hawking, Commun. Math. Phys. 25, 167 (1972).

[70] J. D. Bekenstein, arXiv:gr-qc/9605059.

[71] T. P. Sotiriou and V. Faraoni, Phys. Rev. Lett. 108, 081103 (2012). 
[72] S. Bhattacharya, K. F. Dialektopoulos, A. E. Romano, and T. N. Tomaras, Phys. Rev. Lett. 115, 181104 (2015).

[73] I. Z. Fisher, Zh. Eksp. Teor. Fiz. 18, 636 (1948).

[74] O. Bergmann and R. Leipnik, Phys. Rev. 107, 1157 (1957).

[75] A. I. Janis, E. T. Newman, and J. Winicour, Phys. Rev. Lett. 20, 878 (1968).

[76] H. A. Buchdahl, Int. J. Theor. Phys. 6, 407 (1972).

[77] M. Wyman, Phys. Rev. D 24, 839 (1981).

[78] K. A. Bronnikov, Acta Phys. Pol. B 4, 251 (1973).

[79] M. Campanelli and C. O. Lousto, Int. J. Mod. Phys. D 02, 451 (1993).

[80] L. Vanzo, S. Zerbini, and V. Faraoni, Phys. Rev. D 86, 084031 (2012).

[81] V. Faraoni, F. Hammad, A. M. Cardini, and T. Gobeil, Phys. Rev. D 97, 084033 (2018).

[82] O.A. Fonarev, Classical Quantum Gravity 12, 1739 (1995).

[83] D. Kastor and J. Traschen, Classical Quantum Gravity 34, 035012 (2017).

[84] V. Faraoni and S. D. Belknap-Keet, Phys. Rev. D 96, 044040 (2017).

[85] A. Banijamali, B. Fazlpour, and V. Faraoni, Phys. Rev. D 100, 064017 (2019).

[86] V. Faraoni, D. K. Çiftci, and S. D. Belknap-Keet, Phys. Rev. D 97, 064004 (2018).

[87] A. B. Nielsen and M. Visser, Classical Quantum Gravity 23, 4637 (2006).

[88] G. Abreu and M. Visser, Phys. Rev. D 82, 044027 (2010).

[89] V. Faraoni, Classical Quantum Gravity 33, 015007 (2016).

[90] A. Giusti and V. Faraoni, Classical Quantum Gravity 37, 195005 (2020).

[91] S. A. Hayward, Phys. Rev. D 53, 1938 (1996).

[92] R. L. Arnowitt, S. Deser, and C. W. Misner, Gen. Relativ. Gravit. 40, 1997 (2008).

[93] J. Garcia-Bellido and D. Wands, Phys. Rev. D 53, 5437 (1996).

[94] A. A. Coley and R. J. van den Hoogen, Phys. Rev. D 62 , 023517 (2000).

[95] D. Wands, N. Bartolo, S. Matarrese, and A. Riotto, Phys. Rev. D 66, 043520 (2002).

[96] T. Q. Do, W. F. Kao, and I. C. Lin, Phys. Rev. D 83, 123002 (2011).

[97] K. Bamba, S. D. Odintsov, and P. V. Tretyakov, Eur. Phys. J. C 75, 344 (2015).

[98] E. Elizalde, S. Nojiri, S. D. Odintsov, D. Saez-Gomez, and V. Faraoni, Phys. Rev. D 77, 106005 (2008).

[99] T. Jacobson, Classical Quantum Gravity 24, 5717 (2007).

[100] H. Bondi and C. W. Kilmister, Am. J. Phys. 28, 508 (1960).

[101] N. Dadhich, arXiv:1511.02241.

[102] M. Barriola and A. Vilenkin, Phys. Rev. Lett. 63, 341 (1989).

[103] E. I. Guendelman and A. Rabinowitz, Phys. Rev. D 44, 3152 (1991).

[104] L. Vanzo, G. Acquaviva, and R. Di Criscienzo, Classical Quantum Gravity 28, 183001 (2011).

[105] A. Davidson, S. Rubin, and Y. Verbin, Phys. Rev. D 86, 104061 (2012).

[106] S. W. Hawking, Commun. Math. Phys. 25, 152 (1972).
[107] S. W. Hawking and G. F. R. Ellis, The Large Scale Structure of Space-Time (Cambridge University Press, Cambridge, England), https://doi.org/10.1017/ CBO9780511524646.

[108] C. Brans and R. H. Dicke, Phys. Rev. 124, 925 (1961).

[109] T. P. Sotiriou and V. Faraoni, Rev. Mod. Phys. 82, 451 (2010).

[110] S. Nojiri, S. D. Odintsov, and V. K. Oikonomou, Phys. Rep. 692, 1 (2017).

[111] S. Nojiri and S. D. Odintsov, eConf C0602061, 06 (2006).

[112] S. Nojiri and S. D. Odintsov, Phys. Rev. D 77, 026007 (2008).

[113] S. Nojiri, S. D. Odintsov, and D. Saez-Gomez, Phys. Lett. B 681, 74 (2009).

[114] S. D. Odintsov and V. K. Oikonomou, Phys. Lett. B 807, 135576 (2020).

[115] S. D. Odintsov and V. K. Oikonomou, Phys. Rev. D 101, 044009 (2020).

[116] S. Nojiri and S. D. Odintsov, Phys. Rep. 505, 59 (2011).

[117] A. M. Nzioki, P. K. S. Dunsby, R. Goswami, and S. Carloni, Phys. Rev. D 83, 024030 (2011).

[118] C. G. Park, J. c. Hwang, and H. Noh, J. Cosmol. Astropart. Phys. 09 (2011) 038.

[119] S. Capozziello, M. De laurentis, and A. Stabile, Classical Quantum Gravity 27, 165008 (2010).

[120] P. K. S. Dunsby, AIP Conf. Proc. 1115, 205 (2009).

[121] N. Goheer, J. Larena, and P. K. S. Dunsby, Phys. Rev. D 80, 061301 (2009).

[122] N. Goheer, R. Goswami, and P. K. S. Dunsby, Classical Quantum Gravity 26, 105003 (2009).

[123] K. Ananda, S. Carloni, and P. K. S. Dunsby, Classical Quantum Gravity 26, 235018 (2009).

[124] S. Carloni, A. Troisi, and P. K. S. Dunsby, Gen. Relativ. Gravit. 41, 1757 (2009).

[125] S. Carloni, K. N. Ananda, P. K. S. Dunsby, and M. E. S. Abdelwahab, arXiv:0812.2211.

[126] K. N. Ananda, S. Carloni, and P. K. S. Dunsby, Springer Proc. Phys. 137, 165 (2011).

[127] N. Goheer, J. A. Leach, and P. K. S. Dunsby, Classical Quantum Gravity 25, 035013 (2008).

[128] K. N. Ananda, S. Carloni, and P. K. S. Dunsby, Phys. Rev. D 77, 024033 (2008).

[129] S. Carloni, P. K. S. Dunsby, and A. Troisi, Phys. Rev. D 77, 024024 (2008).

[130] L. Amendola, R. Gannouji, D. Polarski, and S. Tsujikawa, Phys. Rev. D 75, 083504 (2007).

[131] N. Goheer, J. A. Leach, and P. K. S. Dunsby, Classical Quantum Gravity 24, 5689 (2007).

[132] T. Clifton, Classical Quantum Gravity 24, 5073 (2007).

[133] C. Corda, J. Cosmol. Astropart. Phys. 04 (2007) 009.

[134] J. A. Leach, P. K. S. Dunsby, and S. Carloni, arXiv:gr-qc/ 0702122.

[135] S. Carloni and P. K. S. Dunsby, J. Phys. A 40, 6919 (2007).

[136] T. Clifton and J. D. Barrow, Classical Quantum Gravity 23, 2951 (2006).

[137] S. Capozziello, V. F. Cardone, and A. Troisi, Phys. Rev. D 73, 104019 (2006).

[138] J. A. Leach, S. Carloni, and P. K. S. Dunsby, Classical Quantum Gravity 23, 4915 (2006). 
[139] S. Carloni, P. K. S. Dunsby, S. Capozziello, and A. Troisi, Classical Quantum Gravity 22, 4839 (2005).

[140] T. Clifton and J.D. Barrow, Phys. Rev. D 72, 103005 (2005); 90, 029902(E) (2014).

[141] N. Furey and A. DeBenedictis, Classical Quantum Gravity 22, 313 (2005).

[142] A. Pavlov, Int. J. Theor. Phys. 36, 2107 (1997).

[143] M. Ferraris, M. Francaviglia, and I. Volovich, Classical Quantum Gravity 11, 1505 (1994).

[144] D. Burlankov and A. Pavlov, Int. J. Mod. Phys. A 04, 5177 (1989).

[145] G. Leon and E. N. Saridakis, Classical Quantum Gravity 28, 065008 (2011).

[146] Y. Bisabr, Gravitation Cosmol. 16, 239 (2010).

[147] S. Carloni, Open Astron. J. 3, 76 (2010).

[148] S. Capozziello, M. De Laurentis, and M. Francaviglia, Astropart. Phys. 29, 125 (2008).

[149] C. Corda, Mod. Phys. Lett. A 23, 109 (2008).

[150] S. Capozziello, C. Corda, and M. F. De Laurentis, Phys. Lett. B 669, 255 (2008).

[151] A. Aviles Cervantes and J. L. Cervantes-Cota, AIP Conf. Proc. 1083, 57 (2008).

[152] C. F. Martins and P. Salucci, Mon. Not. R. Astron. Soc. 381, 1103 (2007).

[153] S. Capozziello, V. F. Cardone, and A. Troisi, Mon. Not. R. Astron. Soc. 375, 1423 (2007).
[154] S. Mendoza and Y. M. Rosas-Guevara, Astron. Astrophys. 472, 367 (2007).

[155] R. Saffari and Y. Sobouti, Astron. Astrophys. 472, 833 (2007).

[156] C. Corda, arXiv:0710.2605.

[157] S. Capozziello, S. Carloni, and A. Troisi, Recent Res. Dev. Astron. Astrophys. 1, 625 (2003).

[158] S. Capozziello, Int. J. Mod. Phys. D 11, 483 (2002).

[159] V. Faraoni, Phys. Rev. D 83, 124044 (2011).

[160] J. D. Barrow and T. Clifton, Classical Quantum Gravity 23, L1 (2006).

[161] A. F. Zakharov, A. A. Nucita, F. De Paolis, and G. Ingrosso, Phys. Rev. D 74, 107101 (2006).

[162] R. R. Caldwell, Phys. Lett. B 545, 23 (2002).

[163] R. R. Caldwell, M. Kamionkowski, and N. N. Weinberg, Phys. Rev. Lett. 91, 071301 (2003).

[164] S. Nojiri and S. D. Odintsov, Phys. Rev. D 72, 023003 (2005).

[165] S. Nojiri, S. D. Odintsov, and S. Tsujikawa, Phys. Rev. D 71, 063004 (2005).

[166] A. Melchiorri, L. Mersini-Houghton, C. J. Odman, and M. Trodden, Phys. Rev. D 68, 043509 (2003).

[167] H. Gomes, S. Gryb, and T. Koslowski, Classical Quantum Gravity 28, 045005 (2011).

[168] C. Bejarano, R. Ferraro, and M. J. Guzmán, Eur. Phys. J. C 77, 825 (2017). 